\title{
The necroptosis machinery mediates axonal degeneration in a model of Parkinson disease
}

\author{
Maritza Oñate ${ }^{1,2,3} \cdot$ Alejandra Catenaccio $^{1,2} \cdot$ Natalia Salvadores $^{1,2} \cdot$ Cristian Saquel $^{1,2} \cdot$ Alexis Martinez $^{2,4,5}$. \\ Ines Moreno-Gonzalez ${ }^{6,7} \cdot$ Nazaret Gamez $^{6,7} \cdot$ Paulina Soto $^{2,4,5} \cdot$ Claudio Soto $^{6} \cdot$ Claudio Hetz $^{2,4,5,8}$. \\ Felipe A. Court $\mathbb{D}^{1,2,8}$
}

Received: 9 February 2019 / Revised: 14 July 2019 / Accepted: 6 August 2019 / Published online: 7 October 2019

(c) The Author(s), under exclusive licence to ADMC Associazione Differenziamento e Morte Cellulare 2019

\begin{abstract}
Parkinson's disease (PD) is the second most common neurodegenerative condition, characterized by motor impairment due to the progressive degeneration of dopaminergic neurons in the substantia nigra and depletion of dopamine release in the striatum. Accumulating evidence suggest that degeneration of axons is an early event in the disease, involving destruction programs that are independent of the survival of the cell soma. Necroptosis, a programmed cell death process, is emerging as a mediator of neuronal loss in models of neurodegenerative diseases. Here, we demonstrate activation of necroptosis in postmortem brain tissue from PD patients and in a toxin-based mouse model of the disease. Inhibition of key components of the necroptotic pathway resulted in a significant delay of 6-hydroxydopamine-dependent axonal degeneration of dopaminergic and cortical neurons in vitro. Genetic ablation of necroptosis mediators MLKL and RIPK3, as well as pharmacological inhibition of RIPK1 in preclinical models of PD, decreased dopaminergic neuron degeneration, improving motor performance. Together, these findings suggest that axonal degeneration in PD is mediated by the necroptosis machinery, a process here referred to as necroaxoptosis, a druggable pathway to target dopaminergic neuronal loss.
\end{abstract}

\section{Introduction}

Parkinson's disease (PD) is the second most common neurodegenerative disease. The increase in life span and changes in life style predicts that PD will double over the next generation [1]. The characteristic motor symptoms of

\section{Edited by M. Deshmukh}

Supplementary information The online version of this article (https:// doi.org/10.1038/s41418-019-0408-4) contains supplementary material, which is available to authorized users.

$\triangle$ Claudio Hetz

chetz@med.uchile.cl

$\triangle$ Felipe A. Court

felipe.court@umayor.cl

1 Center for Integrative Biology, Faculty of Sciences, Universidad Mayor, Santiago, Chile

2 FONDAP Geroscience Center for Brain Health and Metabolism, Santiago, Chile

3 Department of Physiology, Faculty of Biological Sciences, Pontificia Universidad Católica de Chile, Santiago, Chile
PD include resting tremor, bradykinesia, rigidity of the limbs, and abnormal gait [2], associated to the loss of dopaminergic neurons in the substantia nigra pars compacta $(\mathrm{SNpc})$ and reduction of dopamine release in the striatum [3]. Several genetic alterations have been identified in rare familial PD cases [4], whereas more than $90 \%$ of the cases are considered idiopathic.

A characteristic feature of PD is a progressive "dying back" process of neuronal loss [5]. PD pathology initiates in the striatal terminals and proceeds in a retrograde fashion to the somas in the SNpc [6-9]. Studies using genetic

4 Biomedical Neuroscience Institute (BNI), Faculty of Medicine, University of Chile, Santiago, Chile

5 Program of Cellular and Molecular Biology, Institute of Biomedical Sciences, University of Chile, Santiago, Chile

6 The Mitchell Center for Alzheimer's Disease, Department of Neurology, McGovern Medical School, The University of Texas Health Science Center at Houston, Houston, TX, USA

7 Networking Research Center on Neurodegenerative Diseases (CIBERNED), Department of Cell Biology, Faculty of Sciences, University of Malaga, Malaga, Spain

8 Buck Institute for Research on Aging, Novato, CA 94945, USA 
manipulation demonstrated that apoptosis inhibition prevents cell death of mesencephalic neurons, without a significant impact in the loss of axons and dopamine depletion in the striatum [10-12]. Axonal degeneration is a regulated process, executed by mechanisms that disassemble axonal structures, which are then engulfed by glial cells and circulating macrophages or microglia [13]. Among the mechanisms of axonal destruction, key components include calcium release from the endoplasmic reticulum, mitochondrial dysfunction, and ROS production [14]. We have recently demonstrated that axonal degeneration triggered by mechanical or toxic insults is commanded by a regulated program that involves components of the necroptosis machinery $[15,16]$.

Necroptosis is a programmed cell death mechanism associated with cell loss in several pathological conditions, such as ischemia-reperfusion injury in the heart, liver injury, viral infection, cancer, and neurodegeneration $[17,18]$. Necroptosis can be initiated by a variety of stimuli [19], including TNF- $\alpha$, Fas, TRAIL, interferons, and activation of TLRs (review in [20]). TNF- $\alpha$-induced necroptosis is the best characterized necroptotic initiator to date. Upon TNFR1 activation by TNF- $\alpha$, complex I is formed by the recruitment of the kinase RIPK1, cIAPs1/2, and adapter proteins TRADD and TRAF1/2 [21]. In this complex, RIPK1 is poly-ubiquitylated by cIAPs [22] activating stress pathways (i.e., MAPK and NF-кB), leading to cell survival and inflammation [23]. Nevertheless, dissociation of complex I leads to the formation of a cytosolic pro-cell death machinery (complex II) or the necrosome complex if caspases are inhibited or absent [24]. Upon necrosome formation, RIPK1 is activated and autophosphorylated, activating RIPK3 which binds to and phosphorylates the pseudokinase MLKL, the most downstream effector of necroptosis [20]. Phosphorylated MLKL oligomerizes and translocates to the plasma membrane, forming pores to directly execute a necrotic form of cell death [24, 25]. Other studies suggested that MLKL interacts with the cation channel TRPM7 [26], leading to abnormal calcium influx, cell swelling and plasma membrane rupture [27]. In addition, it has been demonstrated that MLKL is required for necrosome translocation to the mitochondria to enhance aerobic respiration and mitochondrial ROS production, leading to a metabolic collapse [28].

Recent reports associated the activation of necroptosis to several neurodegenerative conditions (reviewed in [29]). Genetic or pharmacological inhibition of necroptosis exert neuroprotective effects in models of brain damage, including ischemia [30-32], traumatic injury [33], viral infections [34], retinal damage [35-37], and spinal cord injury [38]. Recent advances in the field have demonstrated the therapeutic potential of inhibiting necroptosis in several neurodegenerative diseases including amyotrophic lateral sclerosis (ALS) [39, 40] multiple sclerosis (MS) [41] and Alzheimer's disease (AD) [42]. Analysis of postmortem brain tissue derived from PD patients indicated increased levels of RIPK1, RIPK3, and MLKL at the SNpc [43]. Inhibition of RIPK1 using the small molecule nec-1s protected dopaminergic neurons on a pharmacological model of PD in vivo [43] and in vitro [44]. Nevertheless, whether necroptosis activation is functionally associated to motor dysfunction and denervation in PD has not been explored.

In this study, we investigated the contribution of the necroptotic pathway to axonal degeneration in dopaminergic neurons in the context of PD. Analysis of human PD postmortem brain tissue and mouse models of the disease indicated the activation of key components of the necroptosis machinery in axons and somas of dopaminergic neurons of the SNpc. Functional assessment indicated that targeting RIPK1 or MLKL significantly reduced axonal degeneration in dopaminergic primary neurons. Genetic ablation of RIPK3 and MLKL, or administration of nec-1s on a mouse model of PD attenuated axonal degeneration, translating into an improvement of motor performance. Our results demonstrate a novel function of the necroptosis machinery in controlling the mechanisms of axonal destruction in PD and suggest that strategies to inhibit necroptosis may have important therapeutic benefits to attenuate neurodegeneration in PD.

\section{Results}

\section{Pharmacological inhibition of the necroptosis machinery delays 6-OHDA-induced neurite degeneration in vitro}

We studied the involvement of necroptosis in PD using primary neuronal cultures from the embryonic mesencephalon, as this region give rise to the $\mathrm{SNpc}$ during brain development. 6-hydroxydopamine (6-OHDA) was used as a relevant neurotoxic PD insult. First, we evaluated the expression and activation of the necroptotic component MLKL. Phosphorylation of MLKL (pMLKL) expression was studied by immunofluorescence in addition with Acetylated Tubulin staining to visualize neurons. We observed that 6-OHDA triggers a clear modification of the pMLKL staining pattern in neurons, from diffuse in control conditions to a punctate pattern in neurites treated with 6OHDA (Fig. 1a-d), suggesting the formation of MLKL oligomers in degenerating neurons. Moreover, neurons were treated with 6-OHDA for 3 and $6 \mathrm{~h}$ and pMLKL was evaluated by western blot. Low basal expression of pMLKL was detected in control conditions after vehicle treatment, which was increased by threefold after $6 \mathrm{~h}$ of 6-OHDA 

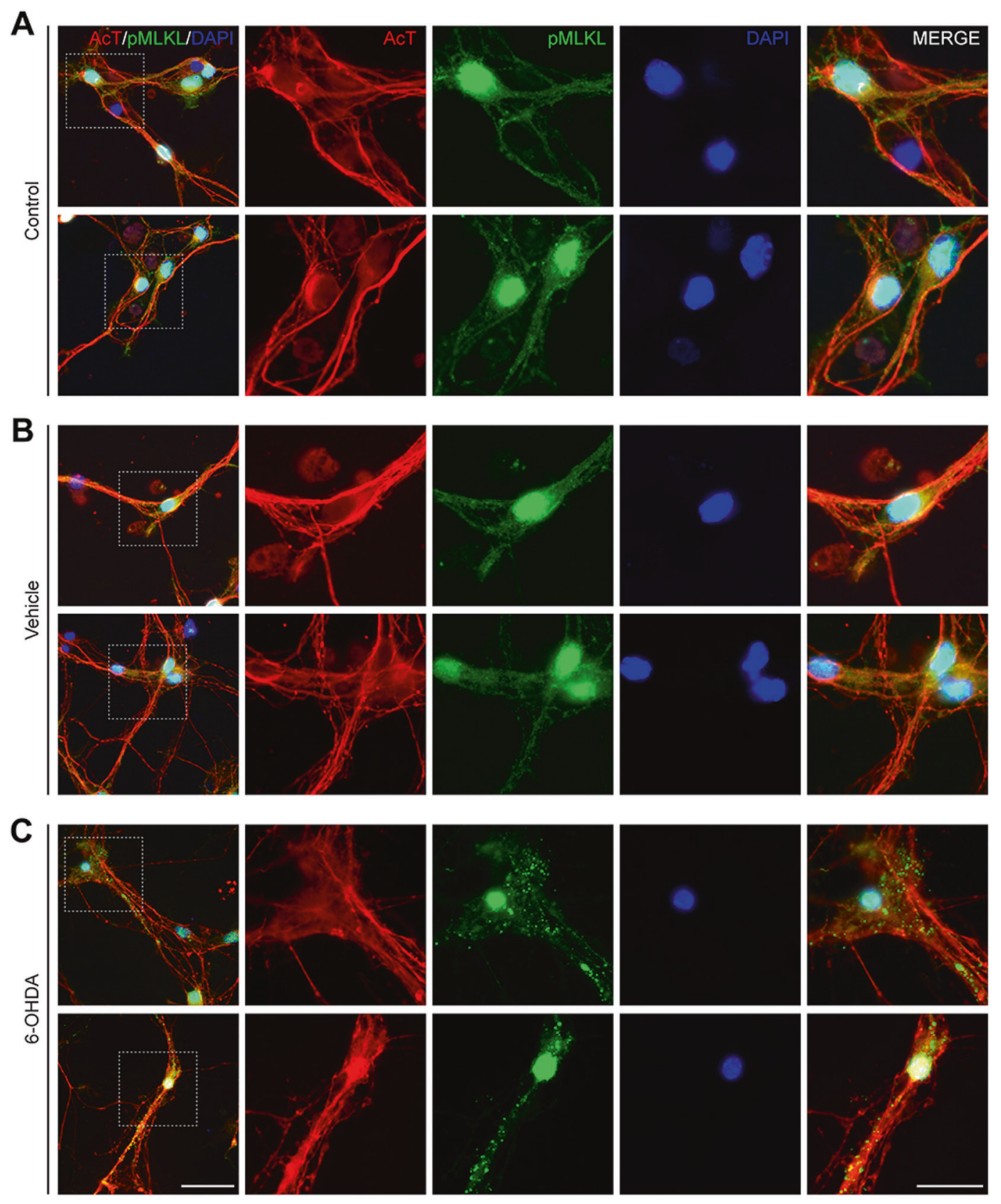

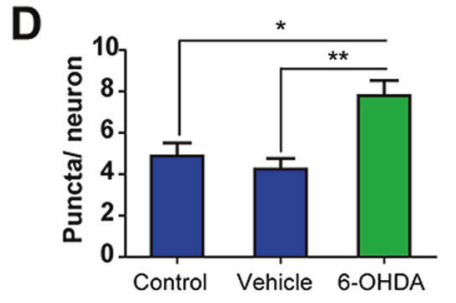

Fig. 1 pMLKL activation in cultured neurons after 6-OHDA treatment. a-c Mesencephalic primary cultures were treated with 6-OHDA or vehicle for $1 \mathrm{~h}$. Untreated cultures were used as control. Cells were immunostained against Acetylated Tubulin (AcT, red) and phosphoMLKL (pMLKL, green). Nuclei were stained with DAPI (blue). d Quantification of the number of pMLKL-positive puncta per neuron was estimated in each condition. e Left, cortical primary cultures were
E

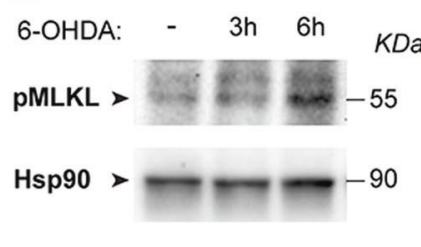

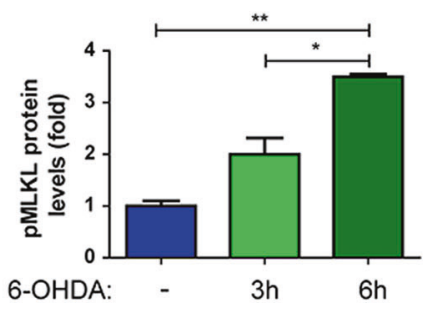

treated with 6-OHDA for 3 or $6 \mathrm{~h}$. pMLKL expression was measured by western blot. Hsp90 was used as loading control. e Right, densitometric analysis was performed in each condition for pMLKL and normalized against Hsp90. Scale bar, $30 \mu \mathrm{m}$; insets, $15 \mu \mathrm{m}$. Data are shown as mean \pm SEM. Statistical differences were obtained using one-way ANOVA followed by Bonferroni's post hoc test. $* p<0.05$, $* * p<0.01 . n=3$ per group 
treatment (Fig. 1e), suggesting activation of this necroptotic component after 6-OHDA treatment.

Then, we manipulated the necroptotic pathway by inhibiting RIPK1 using nec-1s [45]. Mesencephalic neuronal cultures were exposed to 6-OHDA or vehicle as control, in the presence or absence of nec-1s followed by morphological assessment by immunostaining. 6-OHDA induced the degeneration of neurites characterized by fragmentation and neurite beading, a phenomenon that was completely prevented by nec-1s treatment (Fig. 2a). Quantitative assessment of degeneration (see Supplementary Fig. 1A) confirmed the protective effects achieved by nec-1s over neurites (Fig. 2b). Further classification of neurites into intact, beaded, or fragmented indicated almost complete protection of their integrity (Fig. 2c). Of note, nec-1s alone did not alter neurite morphology of mesencephalic neurons (Fig. 2a-c).

Since RIPK1 can trigger apoptotic cell death under certain conditions [46], we studied the participation of MLKL, in 6-OHDA-dependent neurodegeneration. To this end, pharmacological inhibition of MLKL was evaluated in mesencephalic neuronal cultures using GW806742x (GW80), which binds to MLKL blocking its translocation to the plasma membrane [47]. Although GW80 treatment induce a slight, but not significant protection in 6-OHDA treated neurons (Fig. 2d, e and Supplementary Fig. 1B), neurite classification analysis revealed a significant protection of GW80 over 6-OHDA-dependent neurite degeneration process (Fig. 2f).

In addition to dopaminergic neurons, PD has been also associated to neurodegeneration of olfactory, cortical and autonomic peripheral neurons [48]. Therefore, we performed pharmacological inhibition of RIPK1 and MLKL in cortical neuronal cultures exposed to 6-OHDA. Morphologically, treatment with nec-1s or GW80 resulted in a significant protection of neurite degeneration of cortical neurons exposed to 6-OHDA (Fig. $2 \mathrm{~g}-\mathrm{j}$ ).

6-OHDA is known to induce cell death in neurons, indeed treatment with 6-OHDA in mesencephalic and cortical neurons leads to an increase of neurons with condensed
A
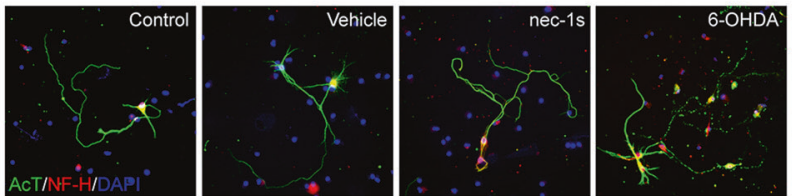

D
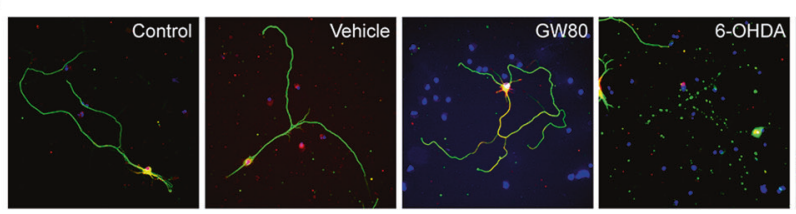

G
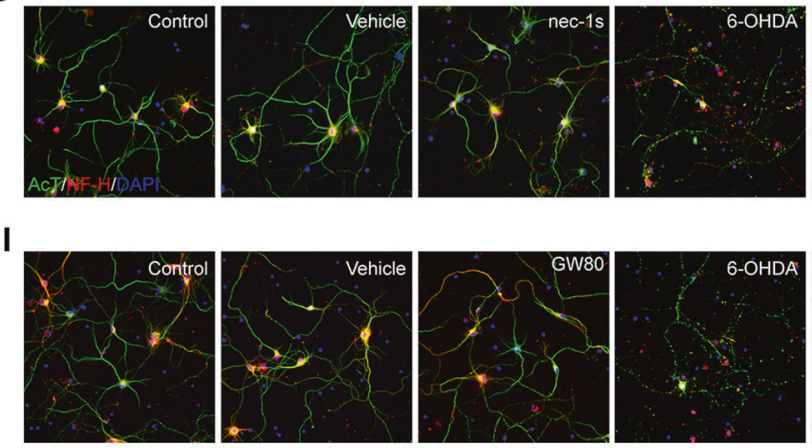

Fig. 2 Pharmacological inhibition of RIPK1 and MLKL delays neurite degeneration in vitro. a-c Mesencephalic neuronal cultures were treated for $6 \mathrm{~h}$ with 6-OHDA alone or together with the RIPK1 inhibitor nec-1s, or vehicle. d-f Similar cultures were treated with 6OHDA alone or together with the MLKL inhibitor GW80, or vehicle. Untreated cultures were used as control. Cells were immunostained for acetylated tubulin (AcT, green) and neurofilament heavy chain (NF-H, red). Nuclei were stained using DAPI (blue). In each condition, the neurite integrity index $(\mathbf{b}, \mathbf{e})$ or a classification of neurite morphology (c, f) was calculated. $\mathbf{g}-\mathbf{h}$ Cortical neuronal cultures were treated for 6

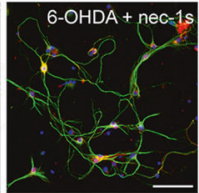

B
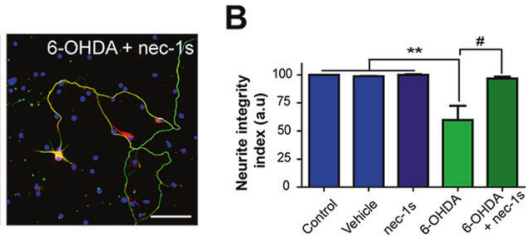

C
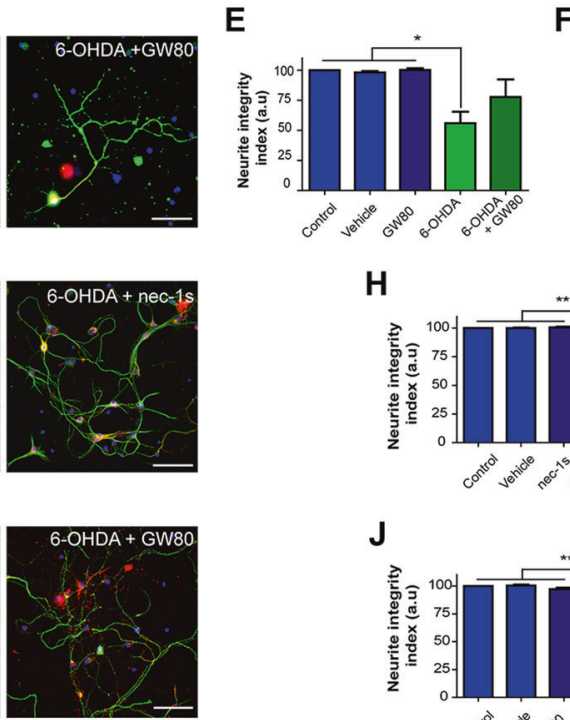

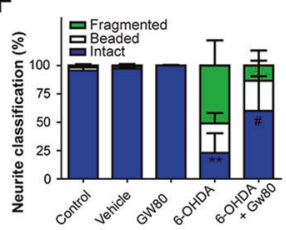

H

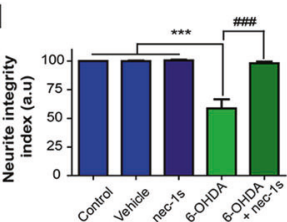

$J$

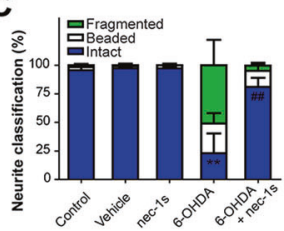

$\mathbf{F}$

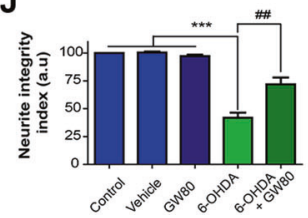

$\mathrm{h}$ with 6-OHDA alone or together with vehicle or the RIPK1 inhibitor nec-1s. i-j Similar cultures were treated with 6-OHDA alone or together with vehicle or the MLKL inhibitor GW80. Cells were stained for acetylated tubulin (green), neurofilament heavy chain (NF-H), and DAPI (blue). $\mathbf{h}, \mathbf{j}$ Neurite integrity index calculated for each condition. Scale bars, $50 \mu \mathrm{m}$. Data are shown as mean \pm SEM. Statistical differences were obtained using one-way ANOVA followed by Bonferroni's post hoc test. $* p<0.05, * * p<0.01 ; * * * p<0.001$ compared with control, vehicle and nec-1s or GW80 conditions. ${ }^{\#} p<0.05$; ${ }^{\# \#} p<0.01$, $\#$ \#\#\# $p<001$ compared with 6-OHDA condition. $n=3$ per group 
nuclei, a morphological reporter of apoptosis, but not a decrease in neuronal density (Supplementary Fig. 2). Nuclei condensation was not protected by RIPK1 or MLKL inhibition using nec-1s or GW80, respectively, suggesting the coactivation of two cell death mechanisms in different neuronal compartments (Supplementary Fig. 2), as we have previously demonstrated for other prodegenerative stimuli [15].

Taken together, our results indicate that treatment with 6OHDA triggers necroptotic components activation. Moreover, pharmacological inhibition of two key components of the necroptotic pathway reduces neurodegeneration in mesencephalic and cortical neurons cultures.

\section{Activation of necroptosis markers in the brain of PD patients}

We next assessed the possible activation of necroptosis markers in the brain of PD patients. We analyzed the phosphorylation levels of MLKL, a measure of its activation, in postmortem samples derived from PD patients and age-matched healthy controls (HC). Analysis of
MLKL phosphorylation at Ser358 and neuromelanin pigment positive cells (marker of dopaminergic neurons) in the SNpc indicated extensive MLKL phosphorylation in PD cases (Supplementary Fig. 3A, B, see inset and arrows in PD samples). As expected, control tissue presented marked neuromelanin staining, which was considerably lower in samples from PD patients (Supplementary Fig. 3A, arrowheads), suggesting loss of dopaminergic neurons. We then assessed the expression of pMLKL in different cellular populations. To this end, double immunofluorescence analysis was performed in HC or PD samples using antibodies against tyrosine hydroxylase (TH), GFAP, and Iba1 to stain dopaminergic neurons, astrocytes, and microglia, respectively, together with pMLKL (Fig. 3a-c). In addition to the marked increase in pMLKL in PD patients, colocalization analysis reveal that around $50 \%$ of the pMLKL signal was localized in TH-positive neurons, $20 \%$ in astrocytes and only $5 \%$ in microglia (Fig. 3d). Furthermore, a strong increase in TH/pMLKL double-positive cells was detected in brain tissue derived from PD patients compared with HC subjects (Fig. 3e).

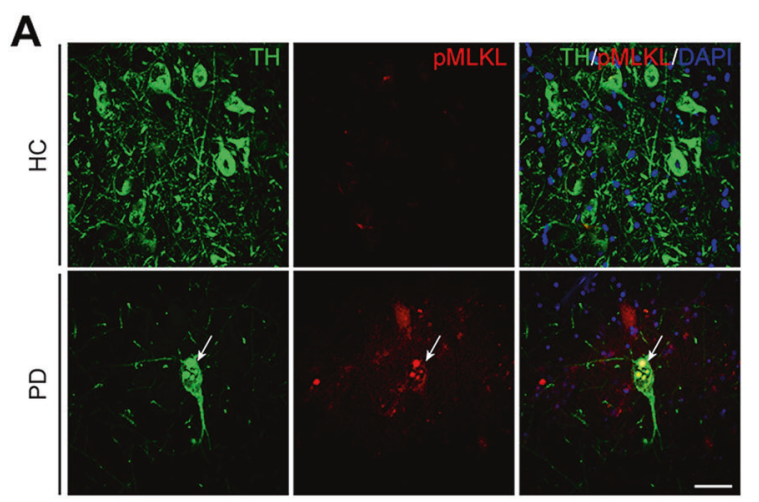

B
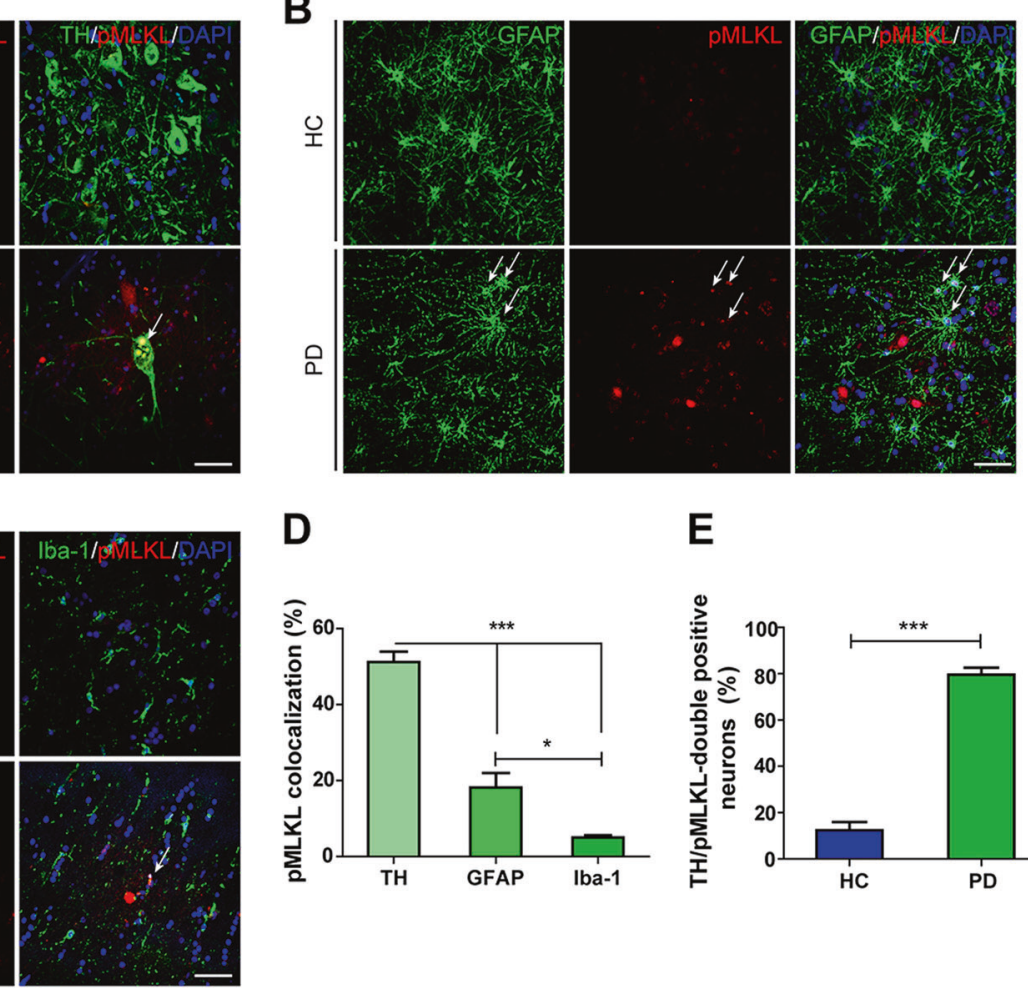

Fig. 3 Activation of pMLKL in postmortem samples of human PD brains. a Human samples of substantia nigra from healthy control patients (HC) and Parkinson's disease patients (PD) were coimmunostained against pMLKL (red) and TH (green), b GFAP (green), and c Ibal (green). Nuclei were stained using DAPI (blue). Arrows indicate double-positive cells. d Percentage of colocalization
D

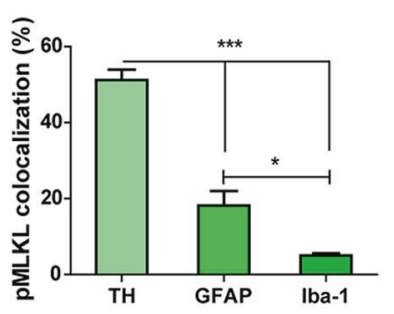

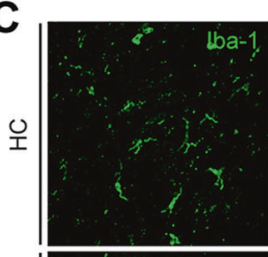
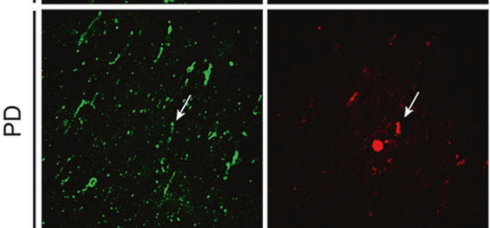

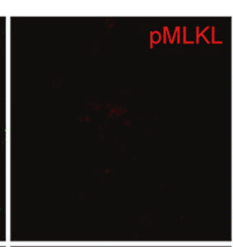
. of pMLKL with different cell markers TH, GFAP and Iba1 was measured in PD samples. e Quantification of TH/pMLKL doublepositive cells in each condition. Scale bars, $25 \mu \mathrm{m}$. Data are shown as mean \pm SEM. Statistical differences were obtained using one-way ANOVA followed by Bonferroni's post hoc test in $\mathbf{d}$ and student's $t$ test in e. ${ }^{*} p<0.05$; $* * p<0.001 . n=3$ per condition 


\section{MLKL activation and necrosome formation in the nigrostriatal pathway after exposure to 6-OHDA}

To establish whether the necroptotic pathway contributes to dopaminergic neuron degeneration in experimental PD, we first analyzed the levels of activation of critical molecular mediators in animals injected with 6-OHDA. To this end, animals were exposed to 6-OHDA in the striatum, and then biochemical and histological analysis was performed in the nigrostriatal circuit (Fig. 4a, b). Western blot analysis indicated elevated levels of phosphorylated MLKL in the striatum only after 3 days of 6-OHDA injection and not at 7 days post treatment (Fig. 4c). Moreover, a transient upregulation of total MLKL levels was observed at 3 days post 6-OHDA injection (Fig. 4c). Similar results were observed for pMLKL in the nigrostriatal pathway (Fig. 4d). Unexpectedly, when the SNpc of the same animals was analyzed, no changes in pMLKL and total MLKL was observed after the 6-OHDA challenge (Fig. 4e).

We then determined if the activation of MLKL occurs on a cell-autonomous manner in dopaminergic neurons. Analyses of the distribution of pMLKL together with TH using co-immunofluorescence revealed a significant increase in pMLKL in dopaminergic neurons at the striatum, nigrostriatal pathway, and SNpc (Fig. 4f-h, upper panel, i). Similar results were obtained when phosphorylated RIPK3 were analyzed in the same regions (Fig. $4 \mathrm{f}-\mathrm{h}$, lower panel). Importantly, the increase in pMLKL and pRIPK3 observed after the 6-OHDA challenge was not present in the respective MLKL-deficient $\left(\mathrm{MLKL}^{-1-}\right.$ ) or RIPK3-deficient mice (RIPK3 ${ }^{-1-}$, Supplementary Fig. 4).

Activation of MLKL by RIPK3 is dependent on the formation of a RIPK1-RIPK3-MLKL necrosome complex [49]. Therefore, formation of the necrosome was evaluated in the striatum 3 days after 6-OHDA injection by immunoprecipitation. Pull down of RIPK1 revealed an increase in pMLKL-RIPK1 interaction in the 6-OHDA injected hemisphere compared with the contralateral side (Fig. 4j). Together, these results demonstrate a progressive and retrograde activation of the necroptosis machinery in a relevant experimental model of PD.

\section{The necroptosis machinery contributes to axonal degeneration and neuronal loss on an animal model of PD}

To study the possible participation of the necroptosis signaling pathway to axonal degeneration in vivo, we set up the experimental conditions to dissociate the process of early axonal degeneration from the loss of somas at the $\mathrm{SNpc}$ on a toxicological model of PD. We established a methodology to evaluate the nigrostriatal circuit in the mouse brain since most of the studies in PD are focused on striatal denervation of axonal terminals in the striatum $(\mathrm{CPu})$ and neuronal cell loss in the SNpc. Serial coronal sections of the entire nigrostriatal circuit of animals unilaterally injected with 6-OHDA at the $\mathrm{CPu}$ were obtained at 3 and 7 days post surgery (Fig. 5a). Striatal denervation was calculated by measuring the optical density of $\mathrm{TH}$ in $\mathrm{CPu}$ sections comparing noninjected with injected hemisphere (Fig. 5b). A significant and progressive decrease was observed at 3 and 7 days after 6-OHDA treatment (Fig. 5c). Axonal degeneration was analyzed along rostro-caudal axis by evaluating axonal tract lengths in both hemispheres (Fig. 5d). In the noninjected hemisphere, axonal tract lengths showed no differences along sections, however a progressive decrease was detected in injected hemispheres 3 and 7 days after 6-OHDA injection (Supplementary Fig. 5A, B). Estimation of the percentage of axonal loss at 3 and 7 days post 6-OHDA injection demonstrated a spatial and temporal progression of axonal degeneration (Fig. 5e). Finally, the loss of dopaminergic neuronal somas was estimated in the SNpc by quantification of TH-positive neurons (Fig. 5f). No changes were observed across the $\mathrm{SNpc}$ at 3 days post 6-OHDA injection, whereas 7 days of treatment resulted in significant neuronal loss (Fig. 5g). These results were also validated when the spatial distribution of TH neurons was quantified (Supplementary Fig. 5C). Injection of vehicle had no effect at the different regions analyzed compared with the noninjected hemisphere (Supplementary Fig. 6A-F). Together, these results showed that the 6-OHDA model is suitable to study axonal degeneration in the absence of evident neuronal loss.

We next performed loss-of-function studies to define the involvement the necroptosis machinery in axonal neurodegeneration and motor impairment triggered by 6-OHDA. To this end, we genetically disrupted the expression of MLKL, as the participation of this protein defines a canonical necroptotic process. $\mathrm{MLKL}^{-1-}$ and their littermate control animals $\left(\mathrm{MLKL}^{+/+}\right)$were injected with 6-OHDA in the right striatum and studied after 7 days. Optical density analysis of the striatum showed no differences in striatal denervation in $\mathrm{MLKL}^{-1-}$ animals when compared with control mice (Fig. 6a, b). In sharp contrast, ablation of MLKL expression significantly protected dopaminergic axonal tracks in animals challenged with 6-OHDA (Fig. 6c, $\mathrm{d}$ and Supplementary Fig. 7A, B). At the SNpc, the number of $\mathrm{TH}$-positive somas were protected in $\mathrm{MLKL}^{-1-}$ mice against 6-OHDA, observing a $21 \%$ of loss when compared with a reduction of $34 \%$ in littermate control animals (Fig. 6e, f and Supplementary Fig. 7C). To validate these results we genetically targeted the expression of RIPK3. $\mathrm{RIPK}^{-1-}$ and their littermate control animals $\left(\mathrm{RIPK}^{+/+}\right.$) were injected with 6-OHDA in the right striatum and 7 days after, serial coronal sections of the entire circuit were obtained to evaluate axonal degeneration. Again, optical 
Fig. 4 Activation of necroptosis in the nigrostriatal pathway after 6-OHDA treatment in vivo.

a Wild-type mice were injected with 6-OHDA in the right striatum and analyzed at 3 or 7 days post injection. b Coronal sections of $2 \mathrm{~mm}$ thickness were obtained from the $\mathbf{i}$ striatum, ii axonal tract, and iii substantia nigra, and the noninjected and injected regions were divided for western blot analysis (indicated in red boxes). pMLKL and MLKL protein expression was evaluated in c striatum,

d nigrostriatal pathway, and e substantia nigra. Hsp90 was used as loading control. Coronal sections of $\mathbf{f}$ striatum,

$\mathbf{g}$ nigrostriatal pathway, and $\mathbf{h}$ substantia nigra from mice injected with 6-OHDA were immunostained at 3 and 7 days post injection. Schemes show the analyzed area in each brain region. (Upper panels) Sections were immunostained for $\mathrm{TH}$ (red) and pMLKL (green) and (Lower panels) TH (red) and pRIPK3 (green). Colocalization in each condition is pointed with arrows. i TH-positive neurons also immunoreactive for pMLKL or pRIPK3 were counted and normalized to the total dopaminergic TH-positive neurons in substantia nigra.

j Proteins extracted from 3 days injected striatum and

contralateral hemisphere were immunoprecipitated with an antibody against RIPK1 and probed for pMLKL. Relative pMLKL levels were calculated by densitometry. Scale bars, 50 $\mu \mathrm{m}$. Data are shown as mean \pm SEM. Statistical differences were obtained using one-way ANOVA followed by Bonferroni's post hoc test in c, $\mathbf{d}, \mathbf{e}$, and $\mathbf{i}$ and by student's $t$-test in j. $* p<0.05 ; * * p<0.01, * * * p$ $<0.001 . n=3$ animals per condition
A

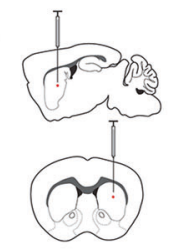

B

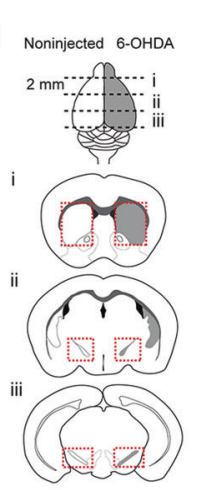

$\mathbf{F}$

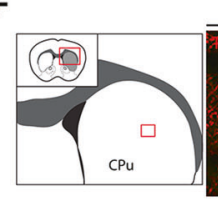

C
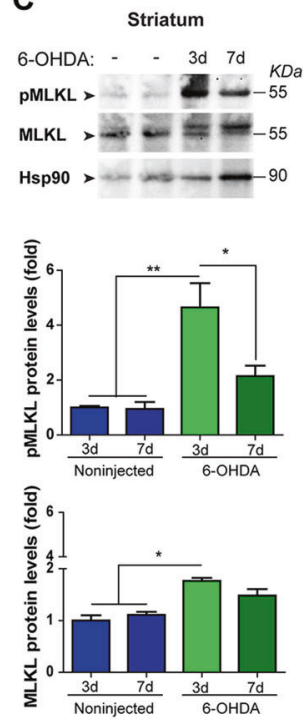

D
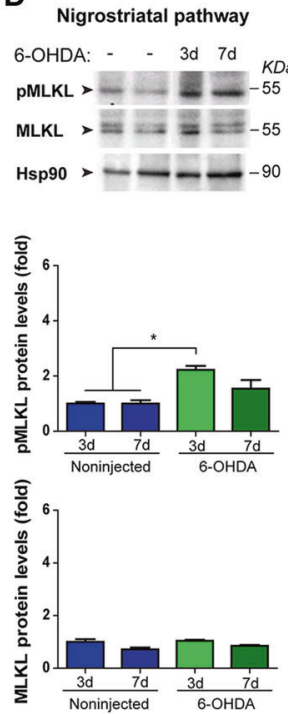

Noninjected
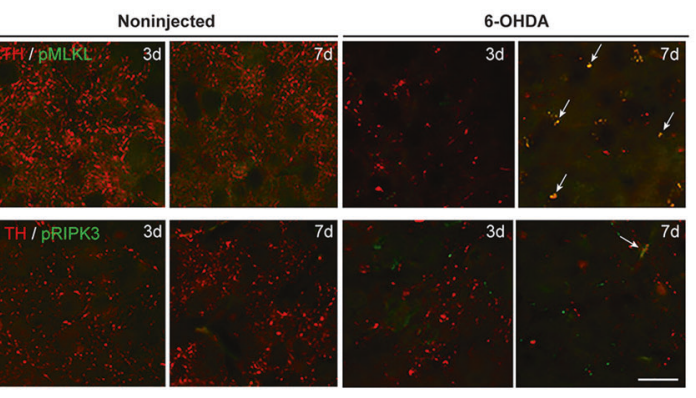

G
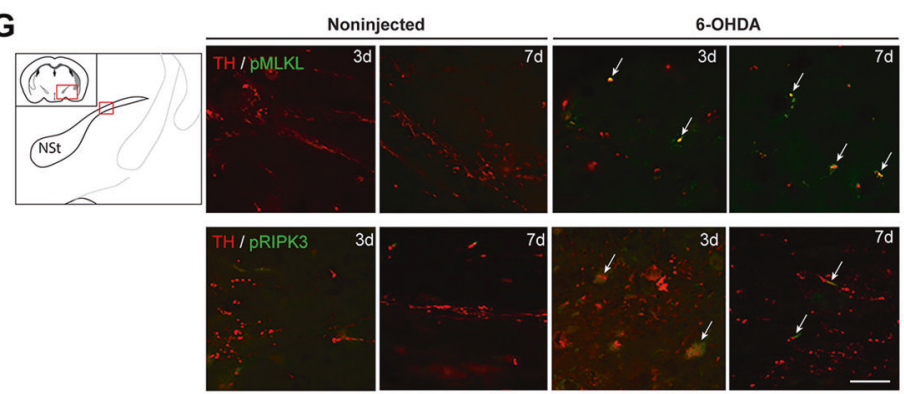

H

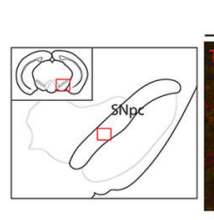

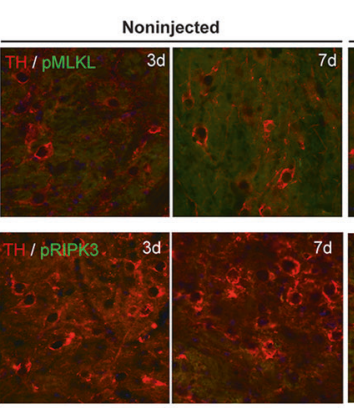

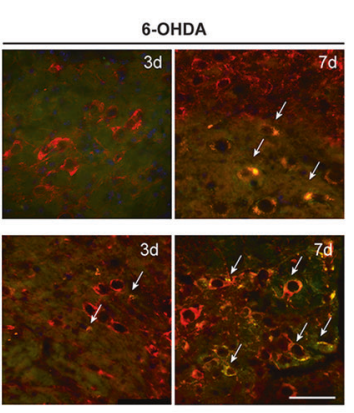

E

Substantia nigra

6-OHDA: - - 3d 7d

PMLKL > - - $--5 s$

MLKL > $\mathrm{m}= \pm=-5$

Hsp90 > - - -9
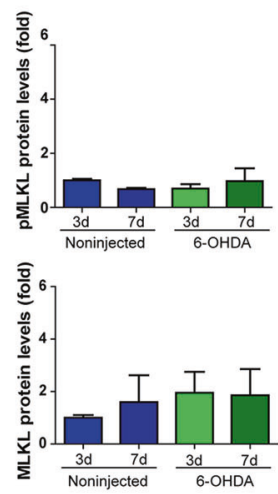

J

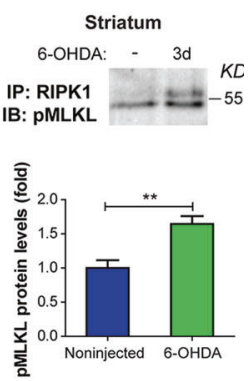

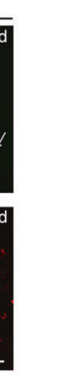

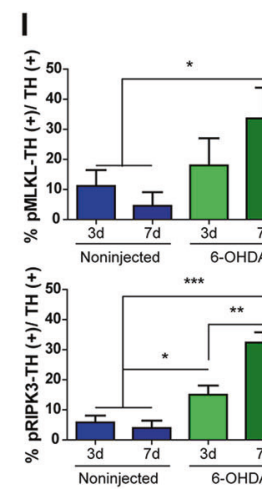

density analysis of the striatum showed no differences in striatal denervation in RIPK $3^{-1-}$ animals when compared with control mice (Fig. 6g, h). In sharp contrast, ablation of
RIP3K expression significantly protected dopaminergic axonal tracks after 6-OHDA (Fig. 6i, j and Supplementary Fig. 7D, E). Together, these results suggest that MLKL and 
A
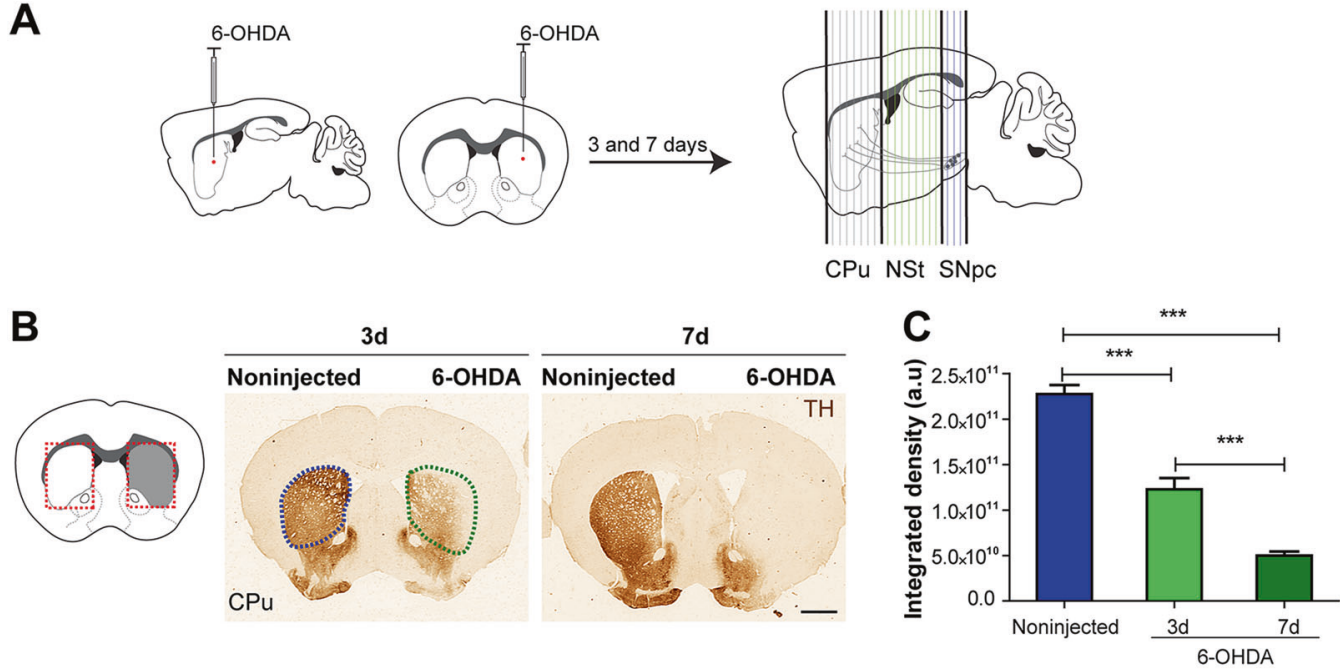

D

$3 d$
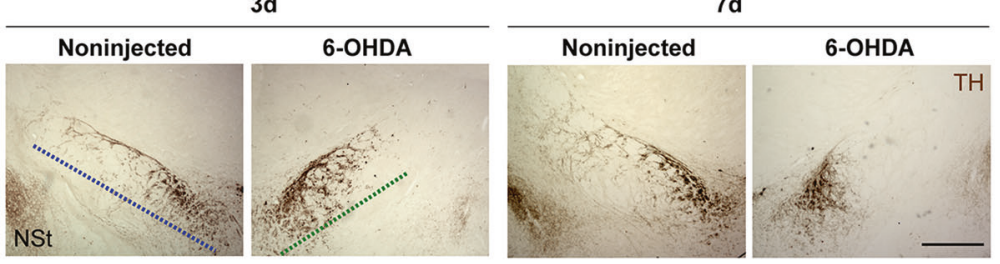

$\mathbf{E}$

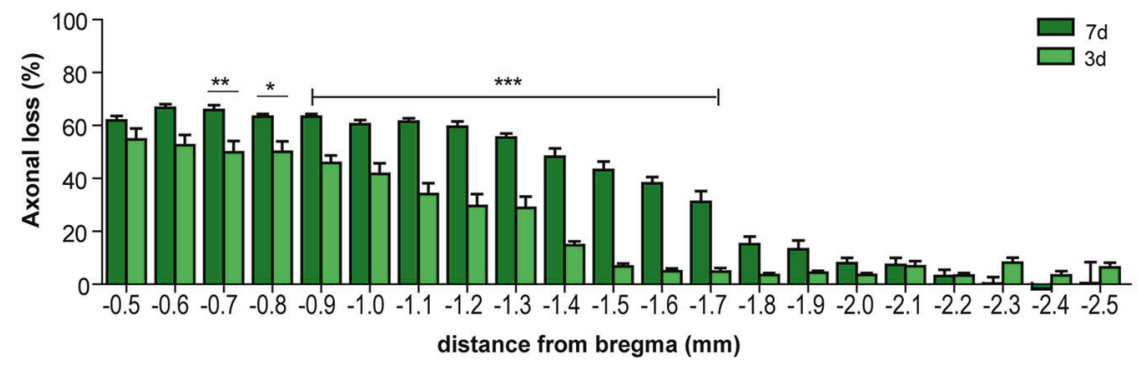

$\mathbf{F}$
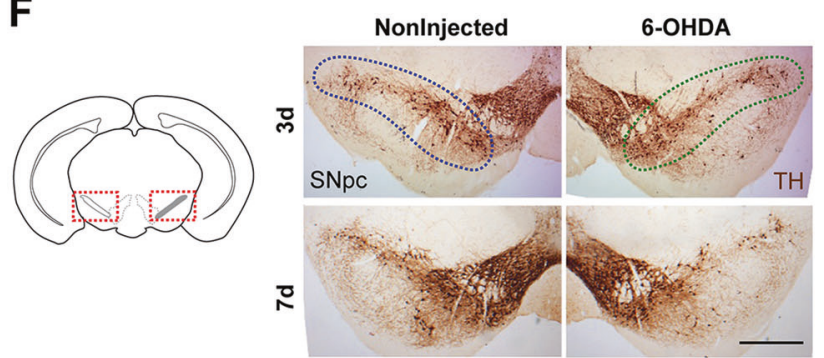

G

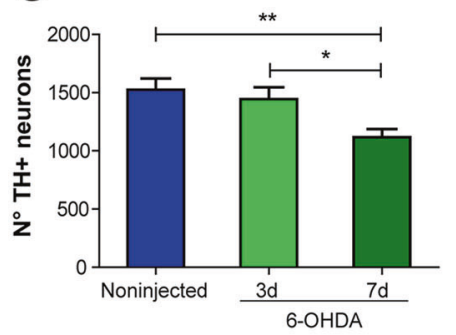

Fig. 5 6-OHDA treatment induces a progressive and retrograde degeneration of nigrostriatal neurons. a Wild-type mice were injected with 6-OHDA in the right striatum. The contralateral hemisphere was kept noninjected as a control. Serial coronal sections of the entire nigrostriatal circuit were obtained at 3 and 7 days post 6-OHDA injection and immunostained for TH. b Left, representative scheme of striatal region analyzed. b Right, the striatum of noninjected and injected hemispheres are demarked with blue and green dashed lines, respectively. Striatal area analyzed $(\mathrm{CPu})$ at 3 and 7 days after injection. Scale bar, $1 \mathrm{~mm}$. c Striatal denervation was calculated as the total integrated optical density in each condition. d Left, scheme of Nigrostriatal (NSt) pathway region analyzed. d Right, NSt at 3 and
7 days post injection. Axonal tracts are indicated using dashed lines for each hemisphere at 3 and 7 days post 6-OHDA injection. Scale bar, $500 \mu \mathrm{m}$. e Spatial distribution of axonal loss at 3 and 7 days post 6OHDA injection. f Left, scheme of the substantia nigra pars compacta (SNpc) region analyzed. f Right, SNpc at 3 and 7 days post 6-OHDA injection. Scale bar, $500 \mu \mathrm{m}$. g Quantification of the total number of TH-positive cells in the entire SNpc at 3 and 7 days post 6-OHDA injection. Data are shown as mean \pm SEM. Statistical differences were obtained using one-way ANOVA in $\mathbf{c}$ and $\mathbf{g}$ and two-way ANOVA in e followed by Bonferroni's post hoc test. *p<0.05; **p $p<0.01$; *** $p$ $<0.001 . n=10$ animals per group 
A

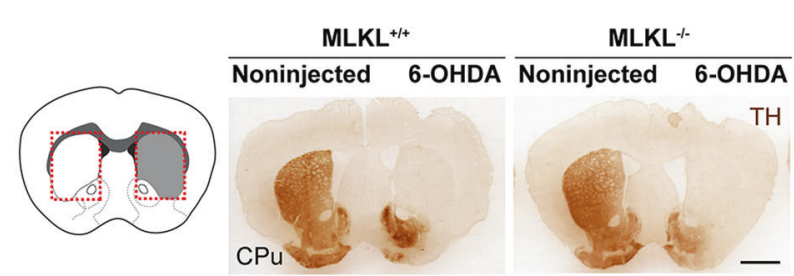

B
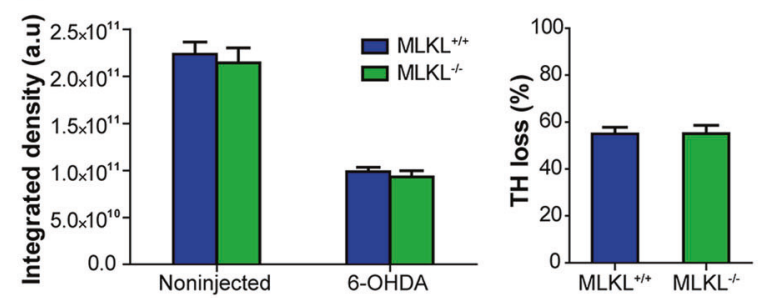

C

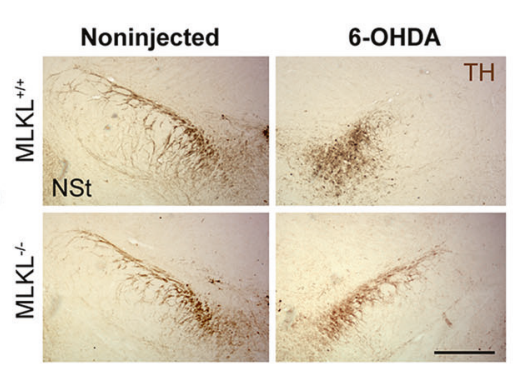

D
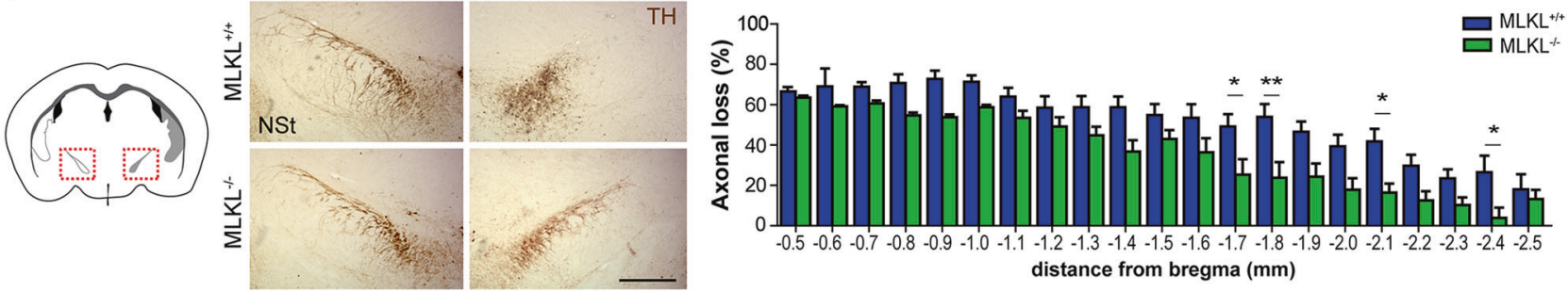

E
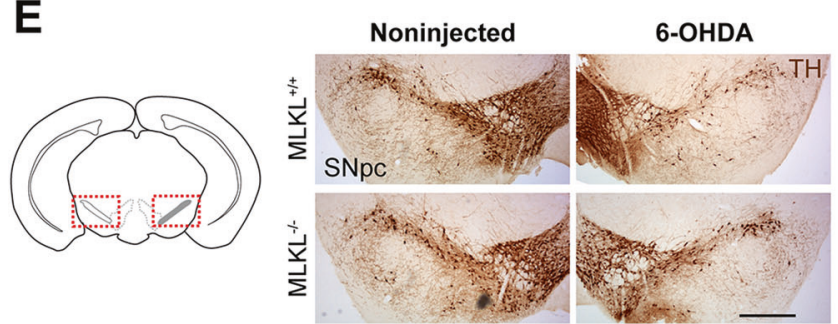

G
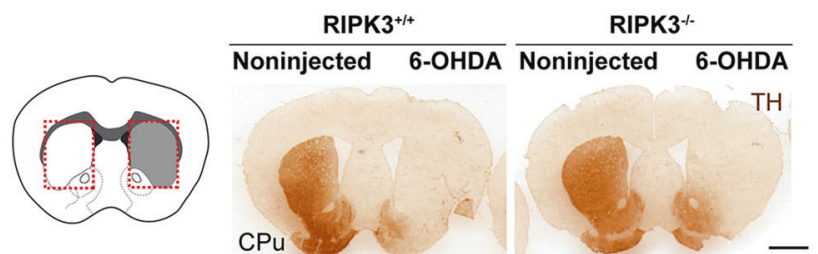

$\mathbf{F}$

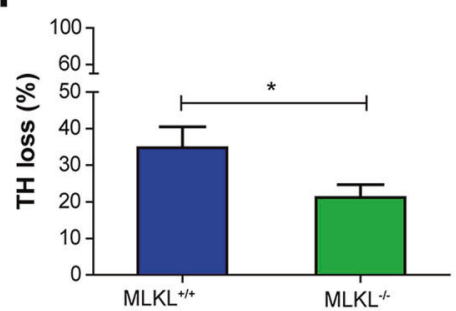

H
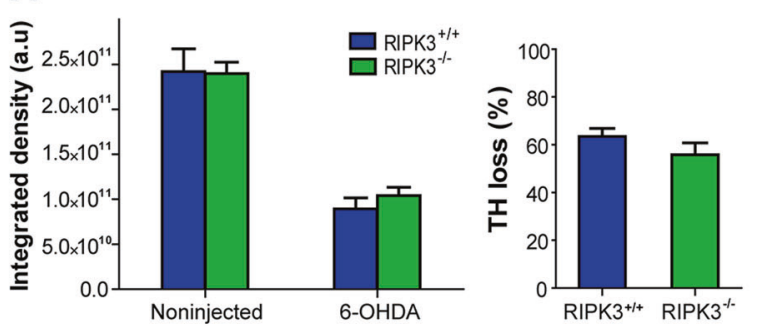

I

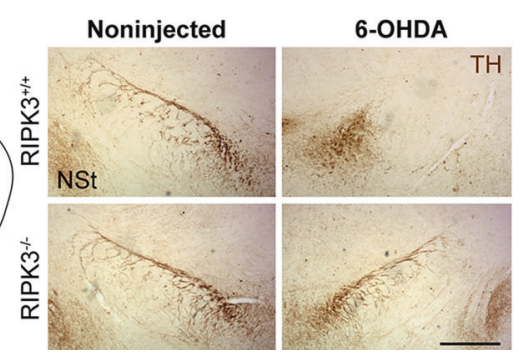

J
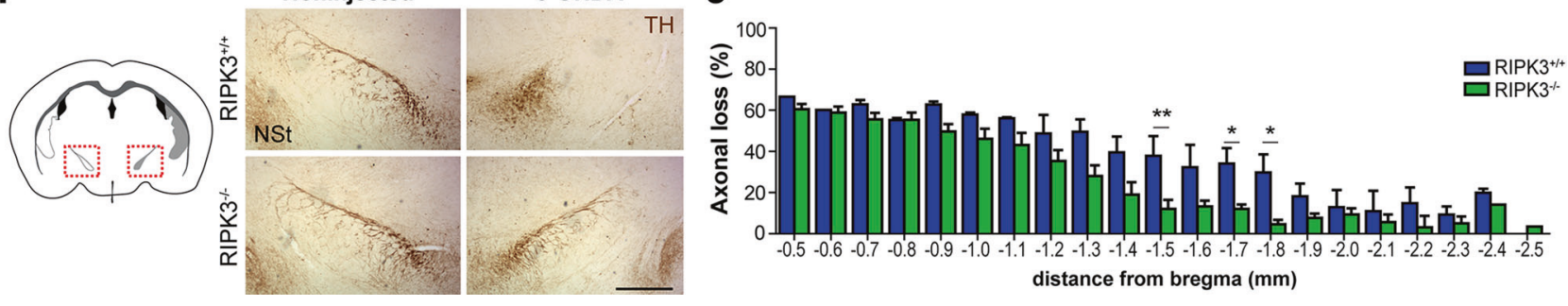

RIPK3 contribute to the dying back degeneration of axons observed in the 6-OHDA model.

\section{Ablation of MLKL and RIPK3 expression improve motor performance on a PD model}

Given that both MLKL and RIPK3 deficiency reduced axonal neurodegeneration induced by 6-OHDA in vivo, we determined if these effects translated in the decrease of motor capacity after 6-OHDA. The cylinder test was performed to measure forepaw akinesia after unilateral 6OHDA lesion. A slight tendency, but no differences were found in $\mathrm{RIPK}^{-1-}$ however, MLKL ${ }^{-1-}$ mice showed a significant improvement in forepaw akinesia at 7 days after injection (Fig. 7a, b). To monitor motor coordination, we performed the rotarod test in the same animals before 
Fig. 6 MLKL and RIPK3 deficiency delays neurodegeneration after 6OHDA injection. $\mathrm{MLKL}^{-1-}$, RIPK $3^{-1-}$, and corresponding WT sibling mice were injected with 6-OHDA in the right striatum $(\mathrm{CPu})$ and the contralateral hemisphere was kept noninjected as a control. Serial coronal sections of the entire nigrostriatal circuit were obtained 7 days after 6-OHDA injection and immunostained for TH. a Left, scheme of striatal region analyzed. a Right, representative striatal coronal sections from $\mathrm{MLKL}^{-1-}$ and MLKL ${ }^{+/+}$mice unilaterally injected with 6OHDA in the right striatum. Scale bar, $1 \mathrm{~mm}$. b Left, striatal denervation was calculated as total integrated optical density in noninjected and injected hemisphere. b Right, percentage of TH loss staining was estimated from integrated density. c Left, scheme of nigrostriatal pathway region analyzed. c Right, representative images from nigrostriatal axons (NSt) from $\mathrm{MLKL}^{-/-}$and $\mathrm{MLKL}^{+/+}$mice injected and immunostained for TH. Scale bar, $500 \mu \mathrm{m}$. d Spatial distribution of axonal loss in each genotype. e Left, scheme of substantia nigra region analyzed. e Right, 6-OHDA induced neuronal loss in $\mathrm{MLKL}^{-1-}$ and $\mathrm{MLKL}^{+/+}$mice analyzed in the substantia nigra pars compacta (SNpc). Scale bar, $500 \mu \mathrm{m}$. f Quantification of total number of THpositive cells in the entire $\mathrm{SNpc}$ represented as the percentage of neuronal loss in each genotype. $\mathrm{g}$ Left, scheme of striatal region analyzed. $\mathrm{g}$ Right, representative striatal coronal sections from RIPK $^{-/-}$and RIPK $^{+/+}$mice injected with 6-OHDA in the right striatum. Scale bar, $1 \mathrm{~mm}$. h Left, striatal integrated density (h, right) and percentage of TH loss calculated in each condition. $\mathbf{i}$ Left, scheme of nigrostriatal pathway region analyzed. i Right, representative coronal sections from nigrostriatal pathway in RIPK $3^{-/-}$and RIPK $3^{+/+}$ injected mice. Scale bar, $500 \mu \mathrm{m}$. j Spatial distribution of axonal loss in each genotype. Data are shown as mean \pm SEM. Statistical differences were analyzed using two-way ANOVA followed by Bonferroni's post hoc test in $\mathbf{b}$ and $\mathbf{h}$, for Integrated density measurements, $\mathbf{d}$ and $\mathbf{j}$, and by student's $t$-test in $\mathbf{b}, \mathbf{f}$ and $\mathbf{h}$, for percentage of loss. * $p<$ 0.05 ; * $p<0.01 . n=8$ animals per group

surgery, and at 3 and 7 days after 6-OHDA injection. No basal alterations in motor performance were detected in both MLKL and RIPK3 null mice (Fig. 7c, d). Remarkably, at 3 days post injection, a reduced decay in performance of about $40 \%$ was observed in $\mathrm{MLKL}^{-1-}$ mice compared with control animals (Fig. 7c). Similarly, improved motor control was observed in RIPK3 knockout (Fig. 7d). Importantly, vehicle injection had no effect in motor performance measured by the rotarod (Supplementary Fig. 6G). Together, these results indicate that the necroptosis machinery mediates in part the neurodegeneration cascade observed in our toxicological model of PD, resulting in improved motor activity.

\section{Pharmacological inhibition of RIPK1 decreases dopaminergic neuron degeneration and reduces motor impairment of experimental PD}

Our previous results indicate a functional role of the necroptosis machinery in the degeneration of dopaminergic axons in vivo. To increase the translational potential of these findings, we tested the consequences of pharmacologically inhibiting the necroptosis machinery in experimental PD. To this end, we intraperitoneally administrated nec-1s daily for 3 days before and after exposing animals to the 6-
OHDA challenge at the striatum. Seven days after 6-OHDA injection morphological analysis of the brain was performed. Consistent with our genetic studies, treatment of animals with nec-1s has no effect in 6-OHDA-dependent striatal denervation (Fig. 8a, b). When the nigrostriatal axonal tract was visualized in the same animals, a significant protection was observed after nec-1s administration (Fig. 8c, d and Supplementary Fig. 8A, B). Furthermore, a significant decrease in forepaw akinesia was found in nec- $1 \mathrm{~s}$ treated mice at 3 days post 6-OHDA injection (Fig. 8e). Finally, motor performance was improved in animals injected with nec-1s (Fig. 8f). Together, these results indicate that pharmacological intervention of the necroptosis machinery results in significant neuroprotection of experimental PD.

\section{Discussion}

PD is a chronic neurodegenerative condition characterized by the degeneration of nigrostriatal dopaminergic neurons located in the SNpc. Axonal loss is emerging as a critical pathological event in PD, which occurs in early stages of the disease and precedes somatic neuronal death [5, 7, 50-52]. In addition, axonal degeneration is a common feature of other diseases including AD, ALS, and MS, representing an interesting transversal target for therapeutic interventions. However, the exact mechanisms by which axons degenerate in PD remains unknown. Here, we unveiled a functional role for RIPK1, RIPK3, and MLKL as part of the molecular machinery that executes axonal degeneration in dopaminergic neurons upon 6-OHDA. Dopaminergic neurons have the largest volume occupied by dendrites and axons, where the volume of the soma is virtually a minor portion of the whole cell [53]. Because of the compartmentalized nature of neurons, the idea that the necroptosis machinery controls the "death" of the axon may have an evolutionary origin where components of necrosome further specialized in this alternative disassembling pathway. Based in our findings, we would like to propose the concept of "necroaxoptosis" as a novel mechanism of axonal degeneration mediated by components of the necroptosis machinery.

Axonal loss in models of PD has been usually quantified using relatively simple measurements [50, 54]. Here, we developed a novel histological method to study of the whole nigrostriatal pathway along the anteroposterior axis. Remarkably, measurements of axonal length in every section were sufficient to depict the retrograde and progressive effect of the 6-OHDA injection along the tract. In addition, biochemical analysis demonstrated a coincident activation of the necroptosis pathway.

Activation of necroptosis has been described in several neurodegenerative conditions [55, 56]. Importantly, 


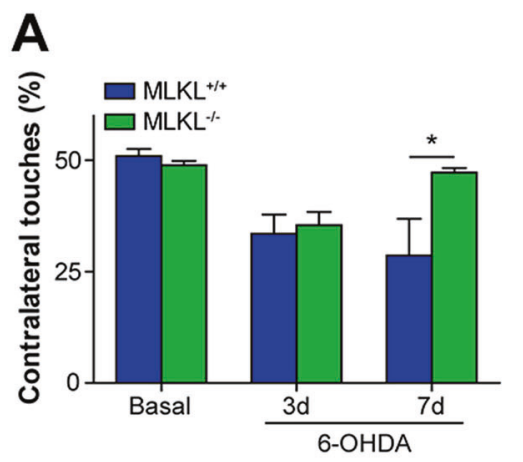

B
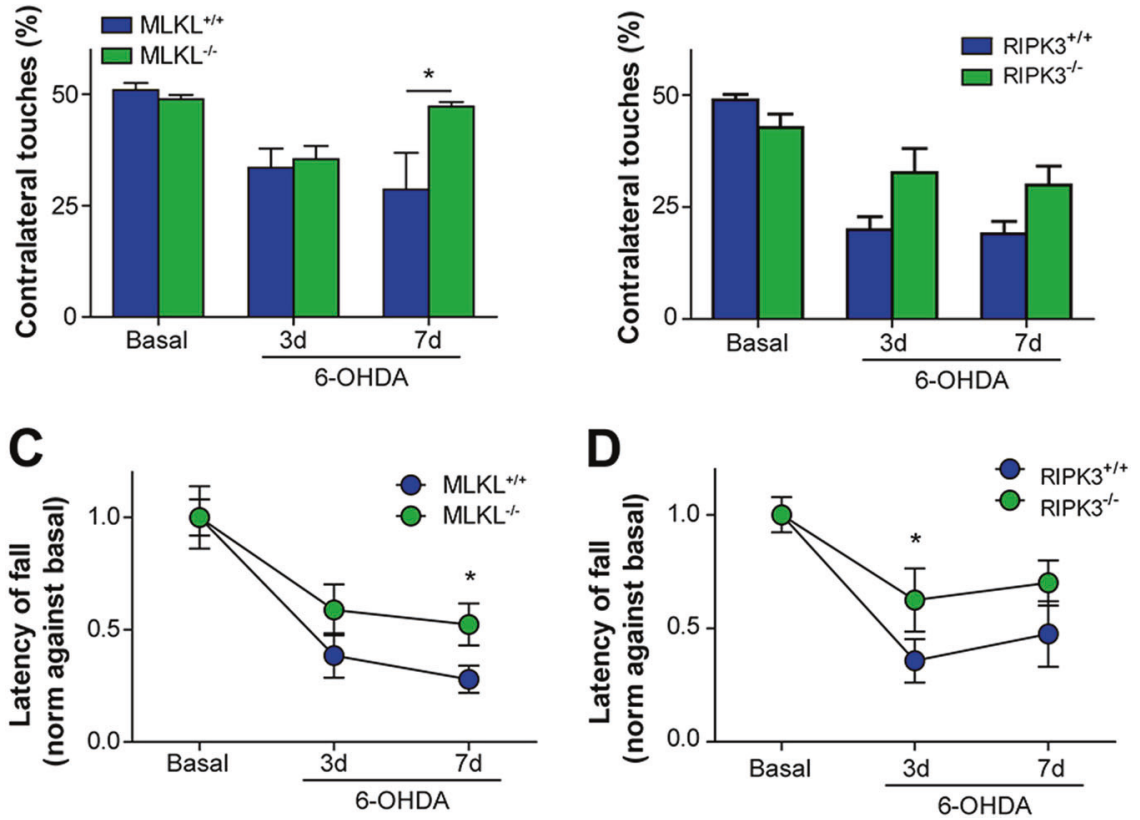

Fig. 7 MLKL and RIPK3 ablation improves motor behavior after 6OHDA injection. MLKL ${ }^{-1-}$, RIPK3 ${ }^{-1-}$ and WT littermate mice were injected with 6-OHDA in the right striatum. Contralateral hemisphere was kept noninjected as a control. a, b Forepaw akinesia after injection was performed using the cylinder test. Percentage of touches from the paw contralateral to the injection side was measured in $\mathrm{MLKL}^{-1-}$ and

RIPK $^{-l-}$, respectively. c, $\mathbf{d}$ Motor performance was tested using the rotarod test by measuring the latency to fall in an accelerated protocol in each genotype. Data are shown as mean \pm SEM. Statistical differences were analyzed using two-way repeated measures ANOVA followed by Bonferroni's post hoc test. $* p<0.05 . n=8$ animals per group

interaction between RIPK1, RIPK3, and MLKL is detected in postmortem samples from MS and AD patients. In our toxicological PD model, we observed an early increase in the interaction between RIPK1 and pMLKL in the striatum, suggesting the formation of the necrosome complex. Whether necroaxoptosis contributes to axonal degeneration in other diseases where necroptosis is important to induce the death of the cell soma remains to be determined.

Previous evidence in neurodegenerative conditions indicates that necroptosis can be induced in glial cells, including astrocytes and oligodendrocytes [39-41]. Our in vitro and in vivo data suggested necroptosis takes place in neurons after treatment with 6-OHDA. Remarkably, our study in human samples showed that pMLKL is mainly expressed in neurons, which is also in agreement with a cell-autonomous activation of necroptosis in the axonal compartment initiated by different prodegenerative stimuli $[15,16]$. Multiple pathological processes may trigger necroptosis in PD, including inflammation and oxidative stress. Its known that $\alpha$-synuclein released from degenerating neurons stimulates microglia, leading to microglia activation and TNF- $\alpha$ secretion $[57,58]$. In agreement with this idea, MPTP-induced striatal dysfunction is reduced in mice lacking TNF- $\alpha$ expression, whereas the loss of dopaminergic neurons at the SNpc is not affected [59]. In other

neurodegenerative conditions, including MS and ALS, sustained inflammation has been associated to TNFomediated necroptosis [29, 60]. Similarly, in a mouse model of ALS, oligodendrocytes showed greater sensitivity to TNFo-induced cell death, and microglia showed increased phosphorylation of RIPK1 and increased expression of a number of inflammatory genes [39]. Therefore, in diverse neurodegenerative conditions, activation of RIPK1 plays a major role in microglia-dependent inflammatory activation, leading to a cell nonautonomous induction of necroptosis in oligodendrocytes and as a consequence, neurodegeneration [60]. Whereas the occurrence of necroptosis in oligodendrocytes across the axon surface contributes to necroaxoptosis remains to be determined.

Overall we propose that the necroptotic machinery operates on a cell-autonomous manner in neurons to execute axonal degeneration possibly involving ROS production as a triggering event [15], a pathological event involved in PD [61-64]. In addition, a cell-nonautonomous mechanism may contribute to this degeneration process in dopaminergic neurons where TNF $\alpha$ might be secreted by microglia. Since the pathways associated to axonal loss are different from those involved in canonical programmed cell death and the destruction of the cell body, necroaxoptosis emerges as a novel and potential mechanism for axonal 
A
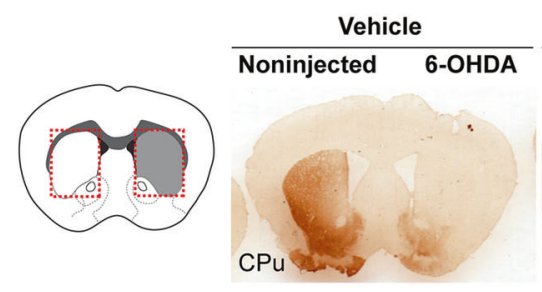

B

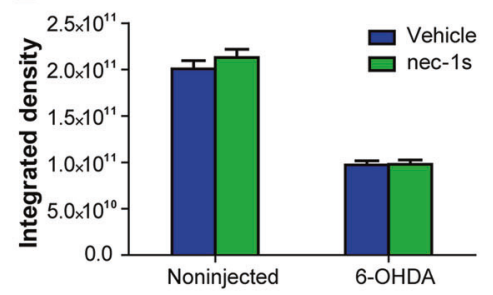

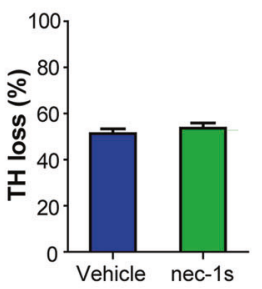

C
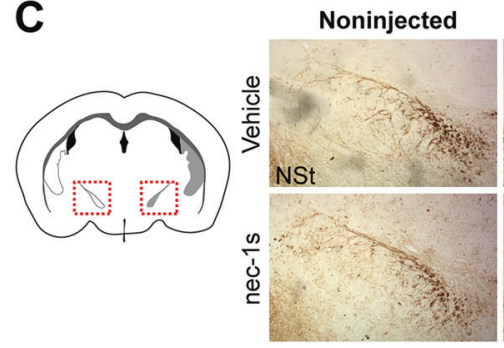

E

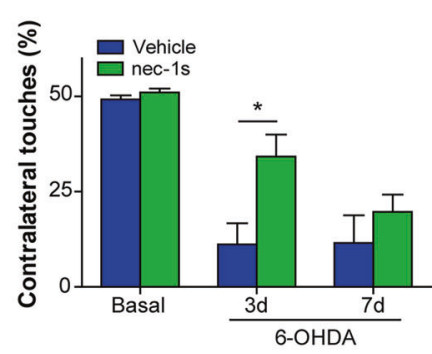

6-OHDA

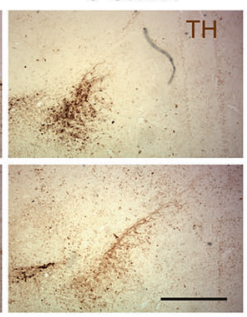

D

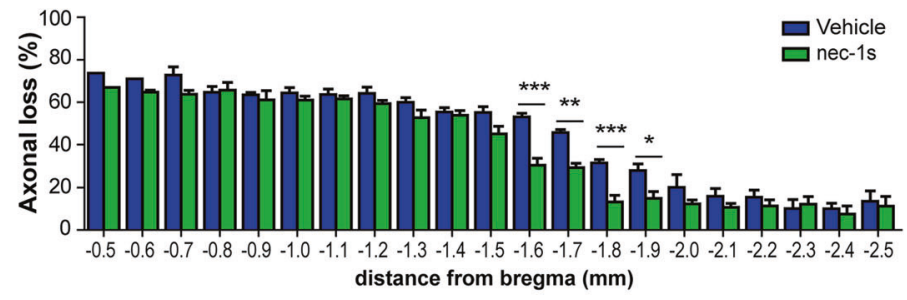

$\mathbf{F}$

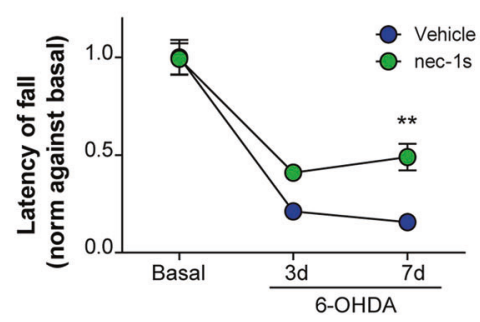

Fig. 8 Pharmacological inhibition of RIPK1 contributes to neurodegeneration and motor impairment after 6-OHDA injection. WT mice were pretreated with nec-1s for 3 days, and then unilaterally injected with 6-OHDA in the right striatum. Mice were followed by 7 days daily injections of nec-1s. Vehicle treatment was used as a control for the nec-1s injections. Left striatum was kept noninjected as control. Serial coronal sections of the entire nigrostriatal circuit were obtained 7 days after 6-OHDA injection and immunostained for TH. a Left, scheme of striatal region analyzed. a Left, representative striatal $(\mathrm{CPu})$ coronal sections from Vehicle or nec-1s treated mice unilaterally injected with 6-OHDA. Scale bar, $1 \mathrm{~mm}$. b Left, striatal denervation 7 days after 6-OHDA injection was calculated as the total integrated optical density in non-injected and injected hemispheres from Vehicle or nec-1s treated mice. b Right, the percentage of TH loss staining was estimated from integrated density. c Left, scheme of nigrostriatal pathway region analyzed. c Right, representative images from nigrostriatal axons (NSt) from WT mice unilaterally injected with 6OHDA and treated with Vehicle or nec-1s. Scale bar, $500 \mu \mathrm{m}$. d Spatial distribution of axonal loss 7 days after 6-OHDA and treated with Vehicle or nec-1s. e Forepaw akinesia was evaluated using the cylinder test. Percentage of touches from the paw contralateral to the injection side was measured. $f$ Motor performance was tested using the rotarod test by measuring the latency to fall in an accelerated protocol. Data are shown as mean \pm SEM. Statistical differences were analyzed using two-way ANOVA followed by Bonferroni's post hoc test in $\mathbf{b}$, left, by student's $t$-test in $\mathbf{b}$, right, and by two-way repeated measures ANOVA followed by Bonferroni's post hoc test in $\mathbf{b}$, c, and $\mathbf{d}$. $* p<$ $0.05 ; * *<0.01 ; * * * p<0.001 . n=9$ animals per group degeneration. Thus, inhibition of necroaxoptosis appears to be a promising therapeutic target for functional and structural preservation of axons and terminals in PD and in other neurodegenerative conditions.

\section{Materials and methods}

\section{Neuronal primary cultures}

Mesencephalic neuronal cultures were obtained from embryonic E14.5 C57BL/6 mice and cortical neuronal cultures were obtained from embryonic E18.5 Sprague-Dawley rats.
The protocol used for both type of primary culture was the same, except the brain structure dissected. Briefly, meninges were removed from each brain and ventral mesencephalon or cortex from both hemispheres were dissected, trypsinized and plated onto $0.1 \mathrm{mg} / \mathrm{ml}$ poly-L-lysine coverslips or plastic dishes. Neurons were grown in Neurobasal media supplemented with B27 and L-glutamine. After 7 days in vitro, neuronal cultures were treated with $40 \mu \mathrm{M}$ of $6-\mathrm{OHDA}$ (in $0.2 \%$ ascorbic acid) or vehicle. For inhibitory treatments, cells were exposed to $30 \mu \mathrm{M}$ of necrostatin-1s (nec-1s, Biovision) or $0.5 \mu \mathrm{M}$ of GW806742x (GW80, AdipoGen). For control conditions, cells were incubated with fresh Neurobasal supplemented medium (control), vehicle or inhibitors alone. 


\section{Puncta quantification analysis}

Quantification of pMLKL puncta in in vitro experiments was performed by measuring the number of pMLKLpositive particles per neuron (based on DAPI and Acetylated Tubulin staining). All images were acquired and processed simultaneously using Image $\mathrm{J}$ software. Binary masks of pMLKL staining images were obtained to analyze size fragment of particles. Particles with a size area between 0.2 and $5 \mu \mathrm{m}^{2}$ were classified as pMLKL-positive puncta.

\section{Neurite integrity index}

Quantification of neurite integrity in in vitro experiments was calculated as the ratio between staining area of intact neurites and total staining area (area of intact neurites + area of fragmented neurites) of Acetylated Tubulin immunofluorescence. Nonneuronal staining was discarded by colocalization of Acetylated Tubulin and Neurofilament heavy chain staining. All images were acquired and processed simultaneously using Image $\mathrm{J}$ software. Binary masks of each image were obtained to analyze size fragment of particles. Particles with a size area equal or lower than $25 \mu \mathrm{m}^{2}$ and with a circularity index higher than 0.3 were classified as degenerated neurite fragments. Particles with a size area higher than $25 \mu \mathrm{m}^{2}$ with a circularity index lower than 0.3 were classified as intact neurite.

\section{Human tissue staining}

Human brain tissue (SNpc) from PD patients $(n=3)$ and control samples (HC, $n=3$ ) was obtained from Banner Sun Health Research Institute. After blocking the endogenous peroxidase activity with $3 \% \mathrm{H}_{2} \mathrm{O}_{2}-10 \%$ methanol for 20 min, brain sections were heated at $80^{\circ} \mathrm{C}$ for $30 \mathrm{~min}$ in 50 $\mathrm{mM}$ citrate buffer $\mathrm{pH} 6.0$ for antigen retrieval prior overnight incubation with anti-phospho-MLKL (S358) mouse monoclonal antibody (1:200; Signalway Antibody). Primary antibody was detected by incubating $1 \mathrm{~h}$ with sheep anti-mouse HRP-linked secondary antibody (General Electric), and peroxidase reaction was visualized using DAB Kit (Vector) following the manufacturer's instructions. Finally, all sections were dehydrated in graded ethanol, cleared in xylene, and cover-slipped with DPX mounting medium. Three samples from each individual were examined under a bright field microscope (DMI6000B, Leica Microsystems) and representative photomicrographs were taken with a digital camera (DFC310 FX Leica). Immunoreactivity percentage was defined as the percentage of area stained with anti-pMLKL related to the substantia nigra analyzed in different coronal sections (three sections/subject). pMLKLimmunopositive signal was converted into 8-bit gray scale and identified by a threshold intensity to quantify the area labeled per total area analyzed. For double immunofluorescence labeling, brain sections were treated with autofluorescence eliminator reagent (2160, Millipore Sigma) and incubated with anti-phospho-MLKL (S358) mouse monoclonal antibody (1:200: Signalway antibody) for $48 \mathrm{~h}$, followed by overnight incubation with secondary primary antibody: rabbit polyclonal anti-TH (1:1000, Millipore Sigma), anti-GFAP (1:1000, Abcam), or anti-Iba1 (1:1000, Wako Chemicals). Sections were sequentially incubated for $1 \mathrm{~h}$ with goat anti-rabbit Alexa 488 and donkey anti-mouse Alexa 594 secondary antibodies (1:500, Invitrogen), rinsed and covered using FluorSave Reagent (Merck Millipore) for immunofluorescent evaluation under a confocal laser microscope (Nikon A1R). Human samples were manipulated following the universal precautions for working with human specimens and as directed by the Institutional Review Board of the University of Texas Health Science Center at Houston (HSC-MS-14-0608).

\section{Experimental animals}

Adult (12-16 weeks old) C57BL/6 mice, MLKL knockout and RIPK3 knockout mice were used. MLKL and RIPK3 knockout mice were kindly provided by Dr Douglas Green (St. Jude Children's Research Hospital, Memphis, TN, USA) and have been described previously $[65,66]$ and have been previously produced and described [67, 68]. Animals were kept under standard conditions of light and temperature and were feed with food and water ad libitum in the Animal Facility of the Sciences Faculty of the Mayor University. The research protocol no. 08-2016 was approved by the Animal Care and Use Scientific Ethic Committee of the Mayor University.

\section{Toxicological model of PD}

Mice were anesthetized with isoflurane and placed in a stereotaxic frame (David Kopf Instruments, USA). A single unilateral injection was performed in the right striatum at the following coordinates: anteroposterior (AP): $+0.07 \mathrm{~cm}$, medio-lateral (ML): $-0.17 \mathrm{~cm}$ and dorso-ventral (DV): $-0.31 \mathrm{~cm}$, relative to bregma (according to [69]) as previously described [70]. Two microliters of a solution of $8 \mu \mathrm{g}$ of 6-OHDA ( $4 \mu \mathrm{g} / \mu \mathrm{l}$ in $0.2 \%$ ascorbic acid) was injected at a rate of $0.5 \mu \mathrm{l} / \mathrm{min}$. As control, we injected $2 \mu \mathrm{l}$ of vehicle solution $(0.2 \%$ ascorbic acid) using the same protocol. Animals were euthanized by overdose of anesthesia at different days post injection for further analysis.

\section{Histological analysis}

Mice were deeply anesthetized with isoflurane and intracardially perfused with isotonic saline followed by $4 \%$ 
paraformaldehyde. Brains were dissected, postfixed overnight in $4 \%$ paraformaldehyde at $4{ }^{\circ} \mathrm{C}$, and then incubated in $30 \%$ sucrose. Tissue was cryoprotected in optimal cutting temperature compound (OCT, Tissue-Tek) at $-20{ }^{\circ} \mathrm{C}$ and serial coronal sections of $25 \mu \mathrm{m}$ thick containing the nigrostriatal circuit (from rostral striatum to ventral midbrain) were obtained using a cryostat (Leica, CM1860). Injected hemisphere was marked for identification. Serial free-floating sections were processed for immunohistochemistry as previously described [70]. Briefly, slices were quenched with $0.3 \% \mathrm{H}_{2} \mathrm{O}_{2}$ for $30 \mathrm{~min}$, blocked with $0.5 \%$ bovine serum albumin and $0.2 \%$ triton $\mathrm{X}-100$ for $2 \mathrm{~h}$ and incubated with primary antibody (rabbit anti-TH, 1:500 Millipore) overnight at $4{ }^{\circ} \mathrm{C}$. Then, sections were washed with $0.1 \mathrm{M}$ PBS and incubated with secondary biotinylated antibody (goat anti-rabbit, 1:500 Vector Laboratories) for $2 \mathrm{~h}$ at RT. After washing, slices were incubated with avidin-biotin-peroxidase complex (Vector Laboratories) for $1 \mathrm{~h}$ at RT followed by $0.1 \mathrm{M}$ PBS washes and developed with 3,3-diaminobenzidine (DAB, SigmaAldrich). Finally, sections were mounted on glass slides with Entellan medium (Merck).

\section{Densitometry analysis of dopaminergic striatal innervation}

Dopaminergic terminals at the striatum $(\mathrm{CPu})$ were analyzed every $100 \mu \mathrm{m}$ of the entire area. Sections were scanned using an Epson Perfection V600 Photo scanner and striatal innervation was quantified by measuring the optical density of TH-immunoreactivity in the striatum using ImageJ software (NIH, USA). Results were expressed as the integrated density of the entire region and as the percentage of TH-immunoreactivity loss compared with control hemisphere as previously described [70].

\section{Quantification of dopaminergic axons}

Dopamine axonal tract at the nigrostriatal pathway (NSt) images were obtained in a Nikon Eclipse E200 microscope. The length of the TH-positive axonal tract was calculated in each section every $100 \mu \mathrm{m}$. Spatial distribution of axonal length was calculated in each section along the anteroposterior axis in injected and contralateral noninjected hemisphere. Percentage of axonal loss was calculated in the injected hemisphere compared with the contralateral hemisphere in each section. Measurements were performed using Image J software.

\section{Dopaminergic neuron cell counting}

Estimation of the number of TH-positive neurons at the SNpc was performed in serial sections of the entire midbrain every $100 \mu \mathrm{m}$ as previously reported [70]. The percentage of $\mathrm{TH}-$ immunoreactive neurons relative to the contralateral (noninjected) side was determined by counting the total number of TH-positive neurons in the entire SNpc. Spatial distribution analysis of each section was performed along anteroposterior axis. Measurements were performed using Image $\mathbf{J}$ software.

\section{Tissue processing and co-immunoprecipitation}

For biochemical analysis, brains were extracted and washed in ice-cold 0.1 M PBS, and then sectioned in a stainless steel brain matrix (coronal slices, $1 \mathrm{~mm}$ spacing). From milimeters sections regions corresponding to the striatum, nigrostriatal axonal pathway, and mesencephalon (containing the entire SNpc) were homogenized in $100 \mu \mathrm{l}$ of RIPA buffer $(50 \mathrm{mM}$ Tris- $\mathrm{HCl}, 150 \mathrm{mM} \mathrm{NaCl}, 1 \% \mathrm{NP}-40,0.5 \%$ Sodium deoxycholate, $0.1 \%$ SDS, and $5 \mathrm{mM}$ EDTA, $\mathrm{pH}$ 7.6) containing protease (1 mM PMSF and protein inhibitor cocktail, PIC) and phosphatase inhibitors (1 $\mathrm{mM} \mathrm{NaF}$ and $50 \mathrm{mM} \mathrm{Na} \mathrm{VO}_{4}$ ). Protein concentration was estimated using the Pierce BCA Protein Assay Kit (Thermo Scientific). For cell culture experiments, cells were collected in $0.1 \mathrm{M}$ PBS, and then homogenated in RIPA buffer for protein quantification as previously described. For immunoprecipitation experiments, $100 \mu \mathrm{g}$ of brain protein lysates were incubated with $4 \mu \mathrm{g}$ of anti-RIPK1 (Cell signaling) for $48 \mathrm{~h}$ while rotating at $4{ }^{\circ} \mathrm{C}$. After incubation, $50 \mu \mathrm{l}$ of Protein A magnetic beads (Invitrogen) were added to each sample and were incubated at $4{ }^{\circ} \mathrm{C}$ for $3 \mathrm{~h}$ while rotating. Following magnetic separation, beads were mixed with loading buffer and boiled at $90^{\circ} \mathrm{C}$ for $5 \mathrm{~min}$. Samples were loaded onto a $10 \%$ SDS/PAGE gel and western blot was performed for pMLKL antibody as followed described.

\section{Western blot}

Brain and cell culture lysates containing $50 \mu \mathrm{g}$ of protein were loaded into $10 \%$ SDS/PAGE gels and transferred onto methanol-activated PVDF membranes (Thermo Scientific). Membranes with transferred proteins were blocked/permeabilized for $1 \mathrm{~h}$ in 5\% BSA in TBS and incubated with different antibodies (mouse anti-pMLKL, 1:1000 Millipore, rabbit anti-MLKL, 1:1000 Abcam, and rabbit anti-Hsp90, 1:1000 Santa Cruz) overnight at $4{ }^{\circ} \mathrm{C}$. Membranes were incubated with HRP-secondary antibodies (goat anti-mouse HRP and goat anti-rabbit HRP, 1:3000 Biorad) for $1 \mathrm{~h}$ at RT and revealed using the ECL (Invitrogen) and Chemidoc ${ }^{\mathrm{TM}}$ MP Imaging System (Biorad). For densitometry analysis of the bands, Image J software was used.

\section{Culture cells immunofluorescence}

For immunocytochemical analysis, neurons were fixed with $4 \%$ paraformaldehyde for $15 \mathrm{~min}$. Then, cells were blocked- 
permeabilized in $5 \%$ gelatin from cold water fish skin and $0.1 \%$ triton $\mathrm{X}-100$ for $1 \mathrm{~h}$ followed by incubation with primary antibodies (mouse anti-acetylated tubulin, 1:1000 Sigma-Aldrich, rabbit anti-Neurofilament heavy chain, 1:500 Sigma-Aldrich, and rabbit anti-pMLKL, 1:250 Abcam) overnight at $4{ }^{\circ} \mathrm{C}$. After washing with $0.1 \mathrm{M}$ PBS, cells were incubated with secondary antibodies (goat antimouse Alexa Fluor 488, goat anti-mouse Alexa Fluor 546, goat anti-rabbit Alexa Fluor 546, and goat anti-rabbit Alexa Fluor 488, 1:1000 Thermo-Fisher) for $2 \mathrm{~h}$ at RT, and then washed with $0.1 \mathrm{M}$ PBS and coverslips were mounted on slides with Fluoromount-G (Electron Microscopy Sciences) solution with DAPI nuclear staining (Thermo-Fisher). Images were obtained using a Leica DMi8 microscope.

\section{Mouse brain immunofluorescence}

For immunofluorescence analysis, $25 \mu \mathrm{m}$ coronal sections from the striatum, nigrostriatal pathway and SNpc were used. Antigen retrieval was performed with boiling Sodium Citrate $10 \mathrm{mM}, \mathrm{pH} 6$ for $10 \mathrm{~min}$ and then, sections were blocked/permeabilized in 5\% BSA, 0,3\% Triton X-100 in $0.1 \mathrm{M}$ TBS for $2 \mathrm{~h}$ at RT, following by primary antibody incubation overnight at $4{ }^{\circ} \mathrm{C}$ (rabbit anti-pRIPK3, 1:200 Abcam; mouse anti-TH, 1:200 Millipore; mouse antipMLKL, 1:200 Millipore; rabbit anti-TH, 1:200 Millipore, in blocking/permeabilizing solution). Sections were washed in $0.1 \mathrm{M}$ TBS and then incubated in secondary antibodies for $2 \mathrm{~h}$ at RT in TBS (goat anti-rabbit Alexa Fluor 488, goat anti-mouse Alexa Fluor 546, goat anti-mouse Alexa Fluor 488, and goat anti-rabbit Alexa Fluor 546, 1:400 ThermoFisher). Finally, sections were washed in 0.1 M TBS and mounted in Mowiol (Sigma).

\section{In vivo necrostatin-1s treatment}

For nec-1s (Biovision, CA, USA) preparation the compound was dissolved in DMSO $(50 \% \mathrm{w} / \mathrm{v})$, and then transferred into 35\% PEG solution as previously described [41]. C57B1/6 mice were treated for 3 days with nec-1s (8 $\mathrm{mg} / \mathrm{kg}$ i.p.) or vehicle before surgery and then, daily injected for 7 days after 6-OHDA injection.

\section{Behavioral tests in mice}

Behavioral tests were performed in injected animals before surgery (baseline) and at 3 and 7 days post injection for analysis of functional motor and coordination performance. The cylinder test was used to assess asymmetric forelimb use as previously reported [70]. Mice were placed in a transparent glass cylinder of $20 \mathrm{~cm}$ diameter for $5 \mathrm{~min}$ and were videotaped during the test. The number of "ipsilateral" (right touches) and "contralateral touches" (left touches) was quantified and represented as the percentage of "contralateral touches" of all movements observed. For the rotarod test, mice were placed into a rotating rod (Model LE8500, Panlab SL) and the time until mice fell was measured (latency fall). Animals were subjected to four trials per day using the accelerated speed test protocol, consisted in increasing speed trial starting with $4 \mathrm{rpm}$ up to $40 \mathrm{rpm}$ within $120 \mathrm{~s}$. Animals waited $\sim 5$ min between each trial to avoid fatigue.

\section{Statistical analysis}

All experiments were analyzed in a blinded manner. Data are shown as mean \pm SEM. Statistical analysis were performed using Student's $t$-test or one-way ANOVA, twoway ANOVA test or two-way repeated measures ANOVA test, followed by Bonferroni's post hoc test or with Mann-Whitney non-parametric test, using GraphPad Prism 5.0 software.

Acknowledgements We are grateful to the Banner Sun Health Research Institute Brain and Body Donation Program of Sun City, Arizona for the provision of brain tissue. This work was supported by Geroscience Center for Brain Health and Metabolism FONDAP15150012 (FAC and CH), Ring Initiative ACT1109 (FAC and CH), FONDECYT-1150766 and FONDECYT-1190518, Canada-Israel Health Research initiative, jointly Funded by the Canadian Institutes of Health Research, the Israel Science Foundation, the international Development Research Centre, Canada and the Azrieli Foundation, Canada (FAC), Conicyt Doctoral Fellowship 21130843 (MO), FONDECYT 1140549 (CH), Millennium Institute P09-015-F (CH), European Commission R\&D MSCA-RISE 734749 (CH). Michael J Fox Foundation for Parkinson's Research-Target Validation grant 9277 (CH), FONDEF ID16I10223 (CH), FONDEF D11E1007 (CH), US Office of Naval Research-Global N62909-16-1-2003 (CH), U.S. Air Force Office of Scientific Research FA9550-16-1-0384 (CH), ALSRP Therapeutic Idea Award AL150111 (CH), Muscular Dystrophy Association $382453(\mathrm{CH})$, and CONICYT-Brazil 441921/ 2016-7 (CH).

Authors Contributions: MO, AC, NS, CS*, IM-G, NG, and PS performed the experiments $\mathrm{MO}, \mathrm{AC}, \mathrm{CS}^{*}, \mathrm{AM}$, and $\mathrm{NG}$ analyzed the data. MO, IM-G, CS**, $\mathrm{CH}$, and FAC designed experiments and wrote the manuscript. $*$ Cristian Saquel and ${ }^{*}$ Claudio Soto.

\section{Compliance with ethical standards}

Conflict of interest The authors declare that they have no conflict of interest.

Publisher's note: Springer Nature remains neutral with regard to jurisdictional claims in published maps and institutional affiliations.

\section{References}

1. Lau L, Breteler M. Epidemiology of Parkinson's disease. Neurol Rev. 2006;5:525-35.

2. Goldman JG, Postuma R. Premotor and nonmotor features of Parkinson's disease. Curr Opin Neurol. 2014;27:434-41. 
3. Dauer W, Przedborski S. Parkinson's disease: mechanisms and models. Neuron. 2003;39:889-909.

4. Corti O, Lesage S, Brice A. What genetics tells us about the causes and mechanisms of Parkinson's disease. Physiol Rev. 2011;91:1161-218.

5. Tagliaferro $P$, Burke RE. Retrograde axonal degeneration in Parkinson disease. J Park Dis. 2016;6:1-15.

6. Kramer ML, Schulz-Schaeffer WJ. Presynaptic alpha-synuclein aggregates, not Lewy bodies, cause neurodegeneration in dementia with Lewy bodies. J Neurosci. 2007;27:1405-10.

7. Kordower JH, Olanow CW, Dodiya HB, Chu Y, Beach TG, Adler $\mathrm{CH}$, et al. Disease duration and the integrity of the nigrostriatal system in Parkinson's disease. Brain. 2013;136:2419-31.

8. Orimo S, Uchihara T, Nakamura A, Mori F, Kakita A, Wakabayashi K, et al. Axonal alpha-synuclein aggregates herald centripetal degeneration of cardiac sympathetic nerve in Parkinson's disease. Brain. 2008;131:642-50.

9. Li Y, Liu W, Oo TF, Wang L, Tang Y, Jackson-Lewis V, et al. Mutant LRRK2(R1441G) BAC transgenic mice recapitulate cardinal features of Parkinson's disease. Nat Neurosci. 2009; $12: 826-8$

10. von Coelln R, Kugler S, Bahr M, Weller M, Dichgans J, Schulz JB. Rescue from death but not from functional impairment: caspase inhibition protects dopaminergic cells against 6hydroxydopamine-induced apoptosis but not against the loss of their terminals. J Neurochem. 2001;77:263-73.

11. Eberhardt O, Coelln RV, Ku S, Rathke-hartlieb S, Gerhardt E, Haid S, et al. Protection by synergistic effects of adenovirusmediated X-chromosome-linked inhibitor of apoptosis and glial cell line-derived neurotrophic factor gene transfer in the 1-methyl4-phenyl-1,2,3,6-tetrahydropyridine model of Parkinson's disease. J Neurosci. 2000;20:9126-34.

12. Ries V, Silva RM, Oo TF, Cheng H-C, Rzhetskaya M, Kholodilov $\mathrm{N}$, et al. JNK2 and JNK3 combined are essential for apoptosis in dopamine neurons of the substantia nigra, but are not required for axon degeneration. J Neurochem. 2008;107:1578-88.

13. Salvadores N, Sanhueza M, Manque P, Court FA. Axonal degeneration during aging and its functional role in neurodegenerative disorders. Front Neurosci. 2017;11:https://doi.org/10. 3389/fnins.2017.00451.

14. Court FA, Coleman MP. Mitochondria as a central sensor for axonal degenerative stimuli. Trends Neurosci. 2012;35:364-72.

15. Hernandez DE, Salvadores NA, Moya-alvarado G, Catalna RJ, Bronfman FC, Court FA. Axonal degeneration induced by glutamate excitotoxicity is mediated by necroptosis. J Cell Sci. 2018;131:https://doi.org/10.1242/jcs.214684.

16. Arrazola MS, Saquel C, Catalan RJ, Barrientos SA, Hernandez $\mathrm{DE}$, Martinez NW, et al. Axonal degeneration is mediated by necroptosis activation. J Neurosci. 2019;39:3832-44.

17. Shan B, Pan H, Najafov A, Yuan J. Necroptosis in development and diseases. Genes Dev. 2003;32:327-40.

18. Tonnus W, Linkermann A. The in vivo evidence for regulated necrosis. Immunol Rev. 2017;277:128-49.

19. Wallach D, Kang T-B, Dillon CP, Green DR. Programmed necrosis in inflammation: toward identification of the effector molecules. Science. 2016;352:aaf2154.

20. Grootjans S, Vanden Berghe T, Vandenabeele P. Initiation and execution mechanisms of necroptosis: an overview. Cell Death Differ. 2017;24:1184-95.

21. Weinlich R, Oberst A, Beere HM, Green DR. Necroptosis in development, inflammation and disease. Nat Rev Mol Cell Biol. 2017;18:127-36.

22. Bertrand MJM, Milutinovic S, Dickson KM, Ho WC, Boudreault A, Durkin J, et al. cIAP1 and cIAP2 facilitate cancer cell survival by functioning as E3 ligases that promote RIP1 ubiquitination. Mol Cell. 2008;30:689-700.
23. Kondylis V, Kumari S, Vlantis K, Pasparakis M. The interplay of IKK, NF- $\kappa B$ and RIPK1 signaling in the regulation of cell death, tissue homeostasis and inflammation. Immunol Rev. 2017;277:113-27.

24. Petrie EJ, Czabotar PE, Murphy JM. The structural basis of necroptotic cell death signaling. Trends Biochem Sci. 2019;44:53-63.

25. Sun L, Wang H, Wang Z, He S, Chen S, Liao D, et al. Mixed lineage kinase domain-like protein mediates necrosis signaling downstream of RIP3 kinase. Cell. 2012;148:213-27.

26. Gong YN, Guy C, Crawford JC, Green DR. Biological events and molecular signaling following MLKL activation during necroptosis. Cell Cycle. 2017;16:1748-60.

27. Cai Z, Jitkaew S, Zhao J, Chiang HC, Choksi S, Liu J, et al. Plasma membrane translocation of trimerized MLKL protein is required for TNF-induced necroptosis. Nat Cell Biol. 2014;16:55-65.

28. Yang Z, Wang Y, Zhang Y, He X, Zhong CQ, Ni H, et al. RIP3 targets pyruvate dehydrogenase complex to increase aerobic respiration in TNF-induced necroptosis. Nat Cell Biol. 2018;20:186-97.

29. Yuan J, Amin P, Ofengeim D. Necroptosis and RIPK1-mediated neuroinflammation in CNS diseases. Nat Rev Neurosci. 2019;20:19-33.

30. Qu Y, Shi J, Tang Y, Zhao F, Li S, Meng J, et al. MLKL inhibition attenuates hypoxia-ischemia induced neuronal damage in developing brain. Exp Neurol. 2016;279:223-31.

31. Yin B, Xu Y, Wei RL, He F, Luo BY, Wang JY. Inhibition of receptor-interacting protein 3 upregulation and nuclear translocation involved in Necrostatin-1 protection against hippocampal neuronal programmed necrosis induced by ischemia/reperfusion injury. Brain Res. 2015;1609:63-71.

32. Zhang S, Wang Y, Li D, Wu J, Si W, Wu Y. Necrostatin-1 attenuates inflammatory response and improves cognitive function in chronic ischemic stroke mice. Medicines. 2016;3:16.

33. You Z, Savitz SI, Yang J, Degterev A, Yuan J, Cuny GD, et al. Necrostatin-1 reduces histopathology and improves functional outcome after controlled cortical impact in mice. J Cereb Blood Flow Metab. 2008;28:1564-73.

34. Bian P, Zheng X, Wei L, Ye C, Fan H, Cai Y, et al. MLKL mediated necroptosis accelerates JEV-induced neuroinflammation in mice. Front Microbiol 2017;8:1-10.

35. Dong K, Zhu H, Song Z, Gong Y, Wang F, Wang W, et al. Necrostatin-1 protects photoreceptors from cell death and improves functional outcome after experimental retinal detachment. Am J Pathol. 2012;181:1634-41.

36. Kim CR, Kim JH, Park HYL, Park CK. Ischemia reperfusion injury triggers $\mathrm{TNF} \alpha$ induced-necroptosis in rat retina. Curr Eye Res. 2016;42:771-9.

37. Viringipurampeer IA, Shan X, Gregory-Evans K, Zhang JP, Mohammadi Z, Gregory-Evans CY. Rip3 knockdown rescues photoreceptor cell death in blind pde6c zebrafish. Cell Death Differ. 2014;21:665-75.

38. Liu M, Wu W, Li H, Li S, Huang LT, Yang YQ, et al. Necroptosis, a novel type of programmed cell death, contributes to early neural cells damage after spinal cord injury in adult mice. J Spinal Cord Med. 2015;38:745-53.

39. Ito Y, Ofengeim D, Najafov A, Das S, Saberi S, Li Y, et al. RIPK1 mediates axonal degeneration by promoting inflammation and necroptosis in ALS. Science. 2016;353:603-8.

40. Re DB, Le Verche V, Yu C, Amoroso MW, Politi KA, Phani S, et al. Necroptosis drives motor neuron death in models of both sporadic and familial ALS. Neuron. 2014;81:1001-8.

41. Ofengeim D, Ito Y, Najafov A, Zhang Y, Shan B, DeWitt JP, et al. Activation of necroptosis in multiple sclerosis. Cell Rep. 2015;10:1836-49. 
42. Caccamo A, Branca C, Piras IS, Ferreira E, Huentelman MJ, Liang WS, et al. Necroptosis activation in Alzheimer's disease. Nat Neurosci. 2017;20:1236-46.

43. Iannielli A, Bido S, Folladori L, Segnali A, Cancellieri C, Maresca A, et al. Pharmacological inhibition of necroptosis protects from dopaminergic neuronal cell death in Parkinson's disease models. Cell Rep. 2018;22:2066-79.

44. Wu JR, Wang J, Zhou SK, Yang L, Le YinJ, Cao JP, et al. Necrostatin-1 protection of dopaminergic neurons. Neural Regen Res. 2015;10:1120-4.

45. Takahashi N, Duprez L, Grootjans S, Cauwels A, Nerinckx W, Duhadaway JB, et al. Necrostatin-1 analogues: critical issues on the specificity, activity and in vivo use in experimental disease models. Cell Death Dis. 2012;3:e437-10.

46. Declercq W, Vanden Berghe T, Vandenabeele P. RIP kinases at the crossroads of cell death and survival. Cell. 2009;138:229-32.

47. Hildebrand JM, Tanzer MC, Lucet IS, Young SN, Spall SK, Sharma $\mathrm{P}$, et al. Activation of the pseudokinase MLKL unleashes the fourhelix bundle domain to induce membrane localization and necroptotic cell death. Proc Natl Acad Sci USA. 2014;111:15072-7.

48. Braak H, Rüb U, Gai WP, Tredici K Del. Idiopathic Parkinson' s disease: possible routes by which vulnerable neuronal types may be subject to neuroinvasion by an unknown pathogen. J Neural Transm (Vienna). 2003;110:517-36.

49. Zhang J, Yang Y, He W, Sun L. Necrosome core machinery: MLKL. Cell Mol Life Sci. 2016;73:2153-63.

50. Cheng HC, Ulane CM, Burke RE. Clinical progression in Parkinson disease and the neurobiology of axons. Ann Neurol. 2010;67:715-25.

51. Chung CY, Koprich JB, Siddiqi H, Isacson O. Dynamic changes in presynaptic and axonal transport proteins combined with striatal neuroinflammation precede dopaminergic neuronal loss in a rat model of AAV-synucleinopathy. J Neurosci. 2009;29:3365-73.

52. Tagliaferro P, Kareva T, Oo TF, Yarygina O, Kholodilov N, Burke RE. An early axonopathy in a hLRRK2(R1441G) transgenic model of Parkinson disease. Neurobiol Dis. 2015;82:359-71.

53. Matsuda W, Furuta T, Nakamura KC, Hioki H, Fujiyama F, Arai $\mathrm{R}$, et al. Single nigrostriatal dopaminergic neurons form widely spread and highly dense axonal arborizations in the neostriatum. $\mathrm{J}$ Neurosci. 2009;29:444-53.

54. West MJ. Estimating length in biological structures. Cold Spring Harb Protoc. 2013;8:412-20.

55. Shao L, Yu S, Ji W, Li H, Gao Y. The contribution of necroptosis in neurodegenerative diseases. Neurochem Res. 2017;42:2117-26.

56. Zhang S, Tang M, Luo H, Shi C, Xu Y. Necroptosis in neurodegenerative diseases: a potential therapeutic target. Cell Death Dis. 2017;8:e2905.
57. Collins LM, Toulouse A, Connor TJ, Nolan YM. Contributions of central and systemic inflammation to the pathophysiology of Parkinson's disease. Neuropharmacology. 2012;62:2153-67.

58. Croisier E, Moran LB, Dexter DT, Pearce RKB, Graeber MB. Microglial inflammation in the parkinsonian substantia nigra: relationship to alpha-synuclein deposition. $\mathbf{J}$ Neuroinflamm. 2005;2:1-8.

59. Ferger B, Leng A, Mura A, Hengerer B, Feldon J. Genetic ablation of tumor necrosis factor-alpha (TNF- $\alpha$ ) and pharmacological inhibition of TNF-synthesis attenuates MPTP toxicity in mouse striatum. J Neurochem. 2004;89:822-33.

60. Wegner KW, Saleh D, Degterev A. Complex pathologic roles of RIPK1 and RIPK3: moving beyond necroptosis. Trends Pharm Sci. 2017;38:202-25.

61. Hsu LJ, Sagara Y, Arroyo A, Rockenstein E, Sisk A, Mallory M, et al. Alpha-synuclein promotes mitochondrial deficit and oxidative. Stress. 2000;157:401-10.

62. Ko L, Mehta ND, Farrer M, Easson C, Hussey J, Yen S, et al. Sensitization of neuronal cells to oxidative stress with mutated human alpha-synuclein. J Neurochem. 2000;75:2546-56.

63. Orth M, Tabrizi SJ, Tomlinson C, Messmer K, Korlipara LVP, Schapira AHV, et al. G209A mutant alpha synuclein expression specifically enhances dopamine induced oxidative damage. Neurochemistry. 2004;45:669-76.

64. Surmeier DJ, Guzman JN, Sanchez-padilla J, Goldberg JA. The origins of oxidant stress in Parkinson's disease and therapeutic strategies. Antioxid Redox Signal. 2011;14:1289-301.

65. Dillon CP, Weinlich R, Rodriguez DA, Cripps JG, Quarato G, Gurung P, et al. RIPK1 blocks early postnatal lethality mediated by caspase-8 and RIPK3. Cell. 2014;157:1189-202.

66. Oberst A, Dillon CP, Weinlich R, McCormick LL, Fitzgerald P, Pop C, et al. Catalytic activity of the caspase-8-FLIPL complex inhibits RIPK3-dependent necrosis. Nature. 2011;471: 363-7.

67. Murphy JM, Czabotar PE, Hildebrand JM, Lucet IS, Zhang JG, Alvarez-Diaz $\mathrm{S}$, et al. The pseudokinase MLKL mediates necroptosis via a molecular switch mechanism. Immunity. 2013; 39:443-53.

68. Newton K, Sun X, Dixit VM. Kinase RIP3 is dispensable for normal NF-kappa Bs, signaling by the B-cell and T-cell receptors, tumor necrosis factor receptor 1 , and Toll-like receptors 2 and 4. Mol Cell Biol. 2004;24:1464-9.

69. Paxinos G, Franklin K. The mouse brain in stereotaxic coordinates. 3rd ed. p. 256. Amsterdam: Academic Press. 2008.

70. Castillo V, Oñate M, Woehlbier U, Rozas P, Andreu C, Medinas D, et al. Functional role of the disulfide isomerase ERp57 in axonal regeneration. PLoS ONE. 2015;10:e0136620. 